\title{
ALMOST DUALITY FOR SAITO STRUCTURE AND COMPLEX REFLECTION GROUPS II: THE CASE OF COXETER AND SHEPHARD GROUPS
}

\author{
YUKIKO KONISHI AND SATOSHI MINABE
}

\begin{abstract}
This article is a sequel to [6]. It is known that the orbit spaces of the finite Coxeter groups and the Shephard groups admit two types of Saito structures without metric. One is the underlying structures of the Frobenius structures constructed by Saito [12] and Dubrovin [4. The other is the natural Saito constructed by Kato-ManoSekiguchi [5] and by Arsie-Lorenzoni [1. We study the relationship between these two Saito structures from the viewpoint of almost duality.
\end{abstract}

\section{INTRODUCTION}

In 1979, K. Saito constructed flat structures on the orbit spaces of the finite Coxeter groups (i.e. the finite real reflection groups) [12]. See also [11. Nowadays, his flat structure is called the Frobenius structure [3]. Generalizing Saito's results, Dubrovin constructed Frobenius structures on the orbit spaces of the Shephard groups [4]. (A Shephard group is the symmetric group of a regular complex polytope.) We call these Frobenius structures the Coxeter-Shephard (or CS) Frobenius structures. Dubrovin gave a characterization of the CS Frobenius structures using his almost duality for Frobenius structures [4].

In this article, we call the Saito structure without metric the Saito structure for short. A Frobenius structure has a Saito structure as an underlying structure [10]. In other words, a Frobenius structure is a Saito structure together with a compatible metric.

There is a distinguished class of finite complex reflection groups called the duality groups. It includes the finite Coxeter groups and the Shephard groups. See [9, §B.4] and also Table 1. Recently, Kato, Mano and Sekiguchi showed the existence of Saito structures on the orbit spaces of the duality groups [5]. Arsie and Lorenzoni also studied the same Saito structures for the duality groups of rank $n=2,3$ [1]. In [6], we formulated the almost duality for the Saito structure and characterized their Saito structure. We call it the natural Saito structure because it comes from the trivial connection.

So the orbit space of a finite Coxeter group or a Shephard group has both the CS Frobenius structure and the natural Saito structure. A natural question is that whether

2010 Mathematics Subject Classification. Primary 53D45; Secondary 20 F55.

Key words and phrases. Frobenius structures, Saito structures, Coxeter groups, Shephard groups. 
the latter is the underlying Saito structure of the former. Arsie and Lorenzoni obtained results on this problem for rank $n=2,3[1]$.

In this article, we revisit the construction of the CS Frobenius structure from the viewpoint of the almost duality of the Saito structure (Theorem 4.4) and show that the multiplication of the natural Saito structure and that of the CS Frobenius structure agree for all the finite Coxeter groups and all the Shephard groups (Corollary 5.3). To prove this, we do not use the classification of these groups explicitly, but use their characterization by degrees (see the condition (CS3) in \$4). We also compare the connections and find that they coincide only for all the finite Coxeter groups and some of the Shephard groups (Theorem 5.4). We need the classification to prove this theorem. In the case of rank $n=2,3$, our results are in accord with [1].

Moreover we find that the natural Saito structure admits a compatible metric if and only if it agrees with the underlying Saito structure of the CS Frobenius structure (Theorem 5.5). Again, the proof does not use the classification explicitly.

The article is organized as follows. In 92 , we first recall the definitions of the Saito structure, the Frobenius structure and the almost duality. Then in 93 , we summarize the natural (almost) Saito structure for the duality groups. In \$4, we explain the CS Frobenius structure for the finite Coxeter groups and the Shephard groups from the viewpoint of the almost duality for Saito structures (Theorem 4.4). \$5 contains the main results of this article, Theorem 5.4 and Theorem 5.5. The remaining sections are devoted to proofs. $\$ 6$ is a preliminary: we write down conditions in the matrix form with respect to flat coordinates of the natural Saito structure. In \$7, we give a proof of Theorem 4.4 and Theorem 5.1. In $₫ 8$, we prove Theorem 5.5, In $\S$ A, we describes some technical details of the example $G(m, 1, n)$.

Acknowledgements. The work of Y.K is supported in part JSPS KAKENHI Kiban-S 16H06337. The work of S.M. is supported in part by JSPS KAKENHI Kiban-C 17K05228.

\section{2. (Almost) Saito structure and (almost) Frobenius structure}

The definition of Saito structure (without metric) can be found in [10]. See also [6] for almost Saito structures.

Definition 2.1. A Saito structure (SS for short) on a manifold $M$ consists of

- a torsion-free flat connection $\nabla$ on $T M$,

- an associative commutative multiplication $*$ on $T M$ with a unit $e \in \Gamma\left(M, \mathcal{T}_{M}\right)$,

- a vector field $E \in \Gamma\left(M, \mathcal{T}_{M}\right)$ called the Euler vector field, 


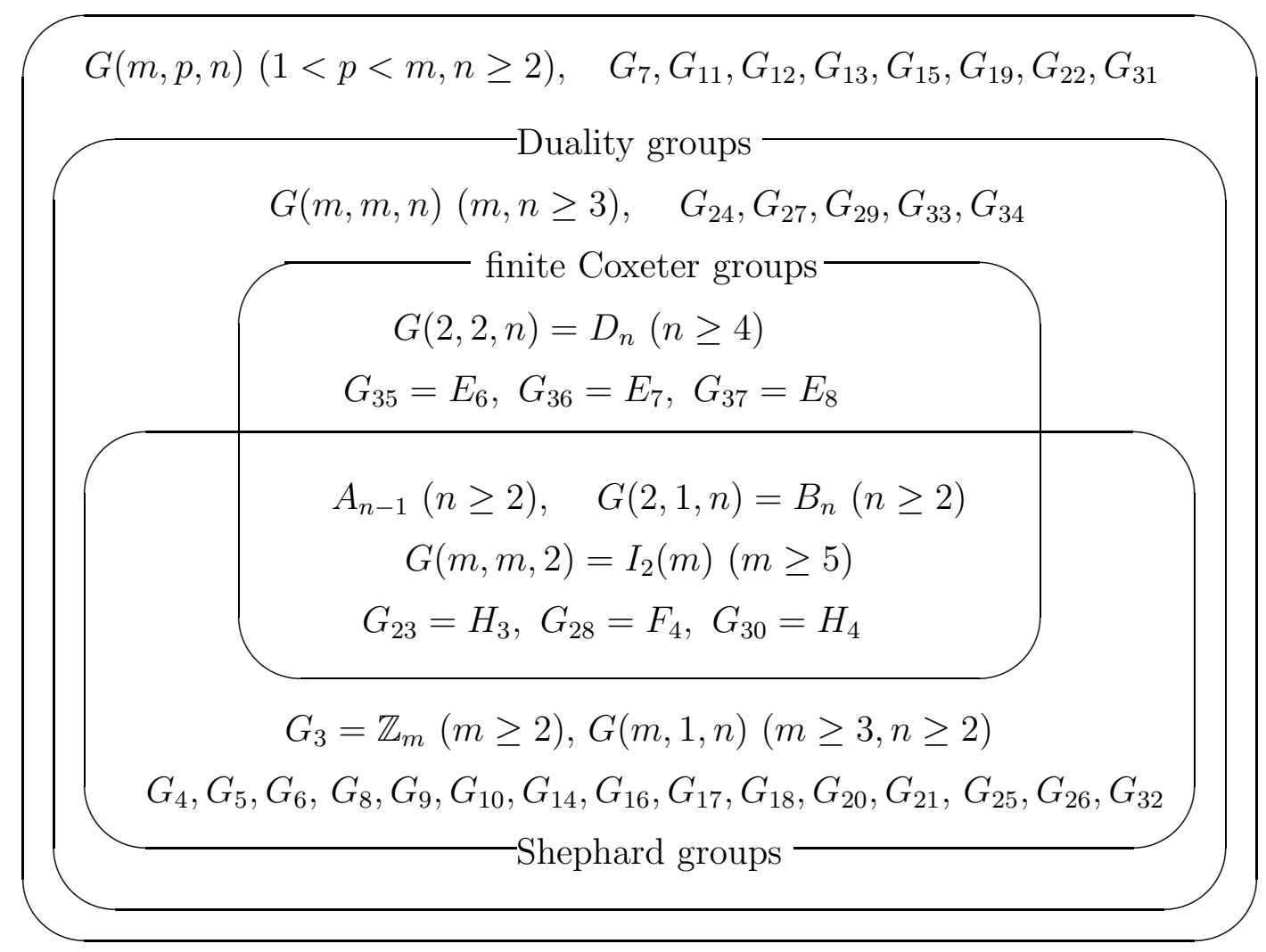

TABLE 1. Irreducible finite complex reflection groups [9, §B.4]. Notations follow [13].

satisfying the following conditions. Let $X, Y, Z \in \mathcal{T}_{M}$ :

(SS1) $\quad \nabla_{X}(Y * Z)-Y * \nabla_{X} Z-\nabla_{Y}(X * Z)+X * \nabla_{Y} Z=[X, Y] * Z$.

(SS2) $\quad[E, X * Y]-[E, X] * Y-X *[E, Y]=X * Y$.

(SS3) $\quad \nabla e=0$.

(SS4) $\quad \nabla_{X} \nabla_{Y} E-\nabla_{\nabla_{X} Y} E=0$.

Definition 2.2. An almost Saito structure (ASS for short) on a manifold $N$ with parameter $r \in \mathbb{C}$ consists of

- a torsion-free flat connection $\boldsymbol{\nabla}$ on $T N$,

- an associative commutative multiplication $\star$ on $T N$ with a unit $E \in \Gamma\left(N, \mathcal{T}_{N}\right)$,

- a nonzero vector field $e \in \Gamma\left(N, \mathcal{T}_{N}\right)$ 
satisfying the following conditions. Let $X, Y, Z \in \mathcal{T}_{N}$ :

(ASS1) $\quad \nabla_{X}(Y \star Z)-Y \star \nabla_{X} Z-\nabla_{Y}(X \star Z)+X \star \nabla_{Y} Z=[X, Y] \star Z$.

(ASS2) $\quad[e, X \star Y]-[e, X] \star Y-X \star[e, Y]+e \star X \star Y=0$.

(ASS3) $\quad \nabla_{X} E=r X$.

(ASS4) $\quad \boldsymbol{\nabla}_{X} \boldsymbol{\nabla}_{Y} e-\boldsymbol{\nabla}_{\boldsymbol{\nabla}_{X} Y} e+\boldsymbol{\nabla}_{X \star Y} e=0$.

There is a following relationship between the Saito structure and the almost Saito structure [6, Proposition 3.7]. Let $(\boldsymbol{\nabla}, \star, e)$ be an ASS on $N$ with the unit $E$ and parameter $r$. For a point $p \in N$, let $\mathcal{P}_{p}=e \star: T_{p} N \rightarrow T_{p} N$ and

$$
N_{0}:=\left\{p \in N \mid \mathcal{P}_{p} \text { is invertible }\right\} .
$$

Then if we define a multiplication $*$ and a connection $\nabla$ by

$$
\begin{aligned}
e \star(X * Y) & =X \star Y, \\
\nabla_{X} Y & =\nabla_{X} Y-\nabla_{X * Y} e,
\end{aligned}
$$

then $e$ is the unit of $*$ and $(\nabla, *, E)$ is a SS on $N_{0}$. Moreover, it holds that

$$
\begin{aligned}
E *(X \star Y) & =X * Y, \\
\nabla_{X} Y & =\nabla_{X} Y+r X \star Y-\nabla_{X \star Y} E .
\end{aligned}
$$

We say that the $\operatorname{SS}(\nabla, *, E)$ is dual to the $\operatorname{ASS}(\boldsymbol{\nabla}, \star, e)$.

Remark 2.3. Given a SS $(\nabla, *, E)$ with the unit $e$, one can make a dual ASS $(\boldsymbol{\nabla}, \star, e)$ with the unit $E$ by (2.3) and (2.4). Notice that there exists a one-parameter family of dual ASS's depending on the choice of the parameter $r \in \mathbb{C}$.

A Frobenius structure [3] on a manifold $M$ of charge $D \in \mathbb{C}$ is a Saito structure $(\nabla, *, E)$ on $M$ together with a nondegenerate symmetric bilinear form ("metric") $\eta$ on $T M$ satisfying the following conditions. Let $X, Y, Z \in \mathcal{T}_{M}$ :

$$
\begin{aligned}
& X(\eta(Y, Z))=\eta\left(\nabla_{X} Y, Z\right)+\eta\left(Y, \nabla_{X} Z\right) . \\
& \eta(X * Y, Z)=\eta(X, Y * Z) . \\
& E \eta(X, Y)-\eta([E, X], Y)-\eta(X,[E, Y])=(2-D) \eta(X, Y) .
\end{aligned}
$$

Note that (2.5) means that $\nabla$ is the Levi-Civita connection of $\eta$ (i.e. the unique torsion free connection on $T M$ compatible with $\eta$ ).

An almost Frobenius structure [4, §3] of charge $D \in \mathbb{C}$ on a manifold $N$ is an almost Saito structure $(\boldsymbol{\nabla}, \star, e)$ with parameter

$$
r=\frac{1-D}{2}
$$


together with a metric $g$ on $T N$ satisfying the following conditions. Let $X, Y, Z \in \mathcal{T}_{N}$ :

$$
\begin{aligned}
& X(g(Y, Z))=g\left(\nabla_{X} Y, Z\right)+g\left(Y, \nabla_{X} Z\right) . \\
& g(X \star Y, Z)=g(X, Y \star Z) . \\
& e g(X, Y)-g([e, X], Y)-g(X,[e, Y])+g(e \star X, Y)=0 .
\end{aligned}
$$

There is a following relationship between the Frobenius structure and the almost Frobenius structure [4]. Let $(g, \star, e)$ be an almost Frobenius structure on $N$ of charge $D$ with the unit $E$. Let us define a multiplication $*$ by (2.1). If we define a metric $\eta$ by

$$
\eta(X, Y)=g(X, E * Y)
$$

then $(\nabla, *, E)$ is a Frobenius structure on $N_{0}$ of the same charge $D$. Moreover, the LeviCivita connections $\nabla$ and $\nabla$ of $g$ and $\eta$ are related by (2.2). We say that $(\eta, *, E)$ is dual to the almost Frobenius structure $(g, \star, e)$.

\section{The natural Saito structure For DUAlity Groups}

3.1. Finite complex reflection groups. For finite complex reflection groups, see [7] and $[9]$.

Let $V=\mathbb{C}^{n}$ and denote by $u^{1}, \ldots, u^{n}$ the standard coordinates of $V$. Let $G$ be a finite complex reflection group acting on $V$. It is well known that the ring of $G$-invariant polynomials $\mathbb{C}[V]^{G}=\mathbb{C}[u]^{G}$ is generated by $n G$-invariant homogeneous polynomials. Such a set of generators $x^{1}, \ldots, x^{n}$ is called a set of basic invariants for $G$. We assume that $x^{1}, \ldots, x^{n}$ are ordered so that the degrees $d_{\alpha}=\operatorname{deg} x^{\alpha}(1 \leq \alpha \leq n)$ are in descending order, i.e.,

$$
d_{1} \geq d_{2} \geq d_{3} \geq \ldots \geq d_{n}
$$

The $\mathbb{C}[V]^{G}$-module of $G$-invariant differential 1 -forms on $V$ is denoted $\Omega_{\mathbb{C}[V]}^{G}$. It is a free $\mathbb{C}[V]^{G}$-module of rank $n$ and $d x^{1}, \ldots, d x^{n}$ form its basis (see [9, Theorem 6.49]). The $\mathbb{C}[V]^{G}$-module of $G$-invariant derivations on $V$ is denoted $\operatorname{Der}_{\mathbb{C}[V]}^{G}$. It is also a free $\mathbb{C}[V]^{G}$ module of rank $n$ (see [9, Lemma 6.48]). A homogeneous basis $\left\{X_{1}, \ldots, X_{n}\right\}$ of $\operatorname{Der}_{\mathbb{C}[V]}^{G}$ is called a set of basic derivations for $G$. The degrees $1 d_{1}^{*}, \ldots, d_{n}^{*}$ of $X_{1}, \ldots, X_{n}$ are called the codegrees of $G$. When necessary, we order $X_{1}, \ldots, X_{n}$ so that the codegrees are in ascending order:

$$
0=d_{1}^{*} \leq d_{2}^{*} \leq \ldots \leq d_{n}^{*}
$$

\footnotetext{
1 In this article, the degree of $\frac{\partial}{\partial u^{\alpha}}$ is counted as $-\operatorname{deg} u^{\alpha}=-1$. If $f \in \mathbb{C}[u]$ is a homogeneous polynomial of degree $d$, the degree of the vector field $f \frac{\partial}{\partial u^{\alpha}}$ is $d-1$.
} 
A polynomial $f \in \mathbb{C}[V]$ defines a homomorphism Hess $(f)$ from the $\mathbb{C}[V]$-module of derivations to the $\mathbb{C}[V]$-module of differential 1 -forms by

$$
\operatorname{Hess}(f)\left(\frac{\partial}{\partial u^{i}}\right)=\sum_{j=1}^{n} \frac{\partial^{2} f}{\partial u^{i} \partial u^{j}} d u^{j} .
$$

If $f$ is $G$-invariant, this homomorphism induces a map from $\Omega_{\mathbb{C}[V]}^{G}$ to $\operatorname{Der}_{\mathbb{C}[V]}^{G}$ (see $[9$, Lemma 6.9]).

Let $M=\operatorname{Spec} \mathbb{C}[V]^{G}=\operatorname{Spec} \mathbb{C}[x] \cong \mathbb{C}^{n}$ be the orbit space of $G$ and let $\pi: V \rightarrow M$ be the orbit map. The complement of reflection hyperplanes is denoted $V^{\circ}$ and its image $\pi(V)$ is denoted $M^{\circ}$. The orbit map $\pi: V^{\circ} \rightarrow M^{\circ}$ is an unbranched covering map. So we can regard the standard coordinates $u^{1}, \ldots, u^{n}$ of $V$ as local coordinates of $M^{\circ}$. We will use the two (local) coordinate systems $x=\left(x^{1}, \ldots, x^{n}\right)$ and $u=\left(u^{1}, \ldots, u^{n}\right)$ on $M^{\circ}$.

Since $\pi: V^{\circ} \rightarrow M^{\circ}$ is locally a homeomorphism, the trivial connection on $T V$ induces a connection $\nabla^{V}$ on $T M^{\circ}$. In the local $u$-coordinates, it is given by

$$
\nabla_{\frac{\partial}{\partial u^{i}}}^{V} \frac{\partial}{\partial u^{j}}=0 \quad(1 \leq i, j \leq n) .
$$

By definition, $\nabla^{V}$ is flat and torsion free.

In this article, we only treat the finite complex reflection groups $G$ which are irreducible (i.e. $G$ acting on $V$ irreducibly) and which satisfy the strict inequality $d_{1}>d_{2}$. The irreducibility implies $d_{\alpha} \geq 2(1 \leq \alpha \leq n)$. The inequality $d_{1}>d_{2}$ implies that the vector field

$$
e:=\frac{\partial}{\partial x^{1}}\left(=\sum_{i=1}^{n} \frac{\partial u^{i}}{\partial x^{1}} \frac{\partial}{\partial u^{i}}\right)
$$

on $M$ is independent of the choice of the set of basic invariants $x^{1}, \ldots, x^{n}$ up to scalar multiplication.2 We also set

$$
E=\frac{1}{d_{1}} E_{\mathrm{deg}}, \quad E_{\mathrm{deg}}=\sum_{i=1}^{n} u^{i} \frac{\partial}{\partial u^{i}}=\sum_{\alpha=1}^{n} d_{\alpha} x^{\alpha} \frac{\partial}{\partial x^{\alpha}} .
$$

\footnotetext{
degree consideration,

$$
\tilde{x}^{1}=a x^{1}+\text { a polynomial in } x^{2}, \ldots, x^{n} \quad(a \in \mathbb{C}, a \neq 0)
$$

and $\tilde{x}^{2}, \ldots, \tilde{x}^{n}$ are polynomials in $x^{2}, \ldots, x^{n}$. Therefore by the chain rule, we have

$$
\frac{\partial}{\partial x^{1}}=\sum_{\alpha=1}^{n} \frac{\partial \tilde{x}^{\alpha}}{\partial x^{1}} \frac{\partial}{\partial \tilde{x}^{\alpha}}=a \frac{\partial}{\partial \tilde{x}^{1}}
$$
}

${ }^{2}$ Assume that $d_{1}>d_{2}$ and that $\tilde{x}=\left(\tilde{x}^{1}, \ldots, \tilde{x}^{n}\right)$ is another set of basic invariants for $G$. Then by 
Notice that the derivation $E_{\operatorname{deg}}$ acts on a homogeneous polynomial $f \in \mathbb{C}[u]$ or $f \in$ $\mathbb{C}[u]^{G}=\mathbb{C}[x]$ as

$$
E_{\operatorname{deg}}(f)=(\operatorname{deg} f) f .
$$

3.2. The natural Saito structure for the duality groups. For an irreducible finite complex reflection group $G$ of rank $n$, the following conditions are equivalent. See e.g. [2, Theorem 2.14].

(D1) $d_{\alpha}+d_{\alpha}^{*}=d_{1}(1 \leq \alpha \leq n)$.

(D2) $G$ is generated by $n$ reflections.

(D3) There exists a set of basic invariants such that the discriminant $\Delta \in \mathbb{C}[x]$ of $G$ is a monic polynomial of degree $n$ as a polynomial in $x^{1}$.

An irreducible finite complex reflection group $G$ satisfying these conditions is called a duality group.

Let $G$ be a duality group and let $x^{1}, \ldots, x^{n}$ be a set of basic invariants for $G$. For a duality group, $d_{1}>d_{2}$ holds. This follows from the classification. Recall that $\nabla^{V}$ given in (3.1) is flat and torsion free. In [6], the followings are proved using the property (D3).

Theorem 3.1. (1) The endomorphism

$$
T_{p} M^{\circ} \rightarrow T_{p} M^{\circ}, \quad X \mapsto \nabla_{X}^{V} e
$$

of the tangent space $T_{p} M^{\circ}$ is invertible at every point $p \in M^{\circ}$ [6. Corollary 7.3]. Therefore the following condition (ASS4) uniquely determines the multiplication $\star$ on $T M^{\circ}$ :

$$
\nabla_{X}^{V} \nabla_{Y}^{V} e-\nabla_{\nabla_{X}^{V} Y}^{V} e+\nabla_{X \star Y}^{V} e=0 \quad\left(X, Y \in \mathcal{T}_{M^{\circ}}\right)
$$

(2) The multiplication $\star$ is associative and commutative and has the unit E.

(3) $\left(\nabla^{V}, \star, e\right)$ is an ASS of parameter $\frac{1}{d_{1}}$ on $M^{\circ}$ [6, Corollary 7.6].

Definition 3.2. $\left(\nabla^{V}, \star, e\right)$ is called a natural ASS for the duality group $G$.

For convenience of the next examples, let us write the statements (1) and (2) in Theorem 3.1 in the $u$-coordinates. Denote the structure constants of the multiplication $\star$ with respect to $\frac{\partial}{\partial u^{1}}, \ldots, \frac{\partial}{\partial u^{n}}$ by $\tilde{B}_{i j}^{k}$, i.e.

$$
\frac{\partial}{\partial u^{i}} \star \frac{\partial}{\partial u^{j}}=\sum_{k=1}^{n} \tilde{B}_{i j}^{k} \frac{\partial}{\partial u^{k}} \quad(1 \leq i, j \leq n) .
$$

Let us set

$$
e^{k}=\frac{\partial u^{k}}{\partial x^{1}}, \quad Q_{j}^{k}=\frac{\partial e^{k}}{\partial u^{j}}
$$


The matrix $Q=\left(Q^{k}{ }_{j}\right)$ is nothing but the representation matrix of the map $X \mapsto \nabla_{X}^{V} e$ with respect to the basis $\frac{\partial}{\partial u^{1}}, \ldots, \frac{\partial}{\partial u^{n}}$. Therefore the statement (1) is equivalent to the condition $\operatorname{det} Q \neq 0$. Eq. (3.5) is equivalent to

$$
\frac{\partial^{2} e^{k}}{\partial u^{i} \partial u^{j}}+\sum_{l=1}^{n} \frac{\partial e^{k}}{\partial u^{l}} \tilde{B}_{i j}^{l}=\frac{\partial Q^{k}{ }_{j}}{\partial u^{i}}+\sum_{l=1}^{n} Q_{l}^{k} \tilde{B}_{i j}^{l}=0 \quad(1 \leq i, j, k \leq n) .
$$

Example 3.3. For $G=\mathbb{Z}_{m}(m \geq 2), n=1, x^{1}=\left(u^{1}\right)^{m}, d_{1}=m$. Therefore

$$
e^{1}=\frac{1}{m\left(u^{1}\right)^{m-1}}, \quad \tilde{B}_{11}^{1}=\frac{m}{u_{1}} .
$$

Example 3.4. For $G=G(m, 1, n)(m \geq 3, n \geq 2)$,

$$
x^{\alpha}=\mathbf{e}_{n+1-\alpha}\left(\left(u^{1}\right)^{m}, \ldots,\left(u^{n}\right)^{m}\right) \quad(1 \leq \alpha \leq n),
$$

where $\mathbf{e}_{\alpha}$ denotes the $\alpha$-th elementary symmetric polynomial. The vector field $e=\frac{\partial}{\partial x^{1}}$ is given by

$$
e=\sum_{k=1}^{n} e^{k} \frac{\partial}{\partial u^{k}}, \quad e^{k}=\frac{(-1)^{n+1}}{m\left(u^{k}\right)^{m-1}} \prod_{\substack{1 \leq j \leq n ; \\ j \neq k}}\left(\left(u^{k}\right)^{m}-\left(u^{j}\right)^{m}\right)^{-1} .
$$

See Corollary A.5. Then it is not difficult to check that the following $\tilde{B}_{i j}^{k}$ 's satisfy (33.6):

$$
\begin{aligned}
& \tilde{B}_{i i}^{i}=\sum_{l \neq i} \frac{m\left(u^{i}\right)^{m-1}}{\left(\left(u^{i}\right)^{m}-\left(u^{l}\right)^{m}\right)}+\frac{m}{u_{i}}, \\
& \tilde{B}_{i i}^{k}=-\frac{m\left(u^{i}\right)^{m-2} u^{k}}{\left(\left(u^{i}\right)^{m}-\left(u^{k}\right)^{m}\right)} \quad(i \neq k) \\
& \tilde{B}_{i j}^{i}=\tilde{B}_{j i}^{i}=-\frac{m\left(u^{j}\right)^{m-1}}{\left(u^{i}\right)^{m}-\left(u^{j}\right)^{m}} \quad(i \neq j) \\
& \tilde{B}_{i j}^{k}=0 \quad(i \neq j \neq k \neq i) .
\end{aligned}
$$

These are the structure constants of $\star$ with respect to the $u$-coordinates.

Now, let $*$ and $\nabla$ be the multiplication and the connection on $T M^{\circ}$ dual to $\star$ and $\nabla^{V}$ (see (2.1) and (2.2)):

$$
e \star(X * Y)=X \star Y, \quad \nabla_{X} Y=\nabla_{X}^{V} Y-\nabla_{X * Y}^{V} e .
$$

Then $(\nabla, *, E)$ is a SS on $M^{\circ}$ and called a natural SS for $G$. Notice that for this case, the converse relations (2.3) and (2.4) become

$$
E *(X \star Y)=X * Y, \quad \nabla_{X}^{V} Y=\nabla_{X} Y+\frac{1}{d_{1}} X \star Y-\nabla_{X \star Y} E .
$$

In [6, Theorem $7.5(3)]$, the following theorem is proved.

Theorem 3.5. The natural $S S(\nabla, *, E)$ for $G$ is polynomial i.e., 
(i) there exists a system of $\nabla$-flat coordinates $t=\left(t^{1}, t^{2}, \ldots, t^{n}\right)$ which is a set of basic invariants for $G$, and

(ii) the structure constants of the multiplication with respect to the basis $\frac{\partial}{\partial t^{1}}, \ldots, \frac{\partial}{\partial t^{n}}$ are polynomials in $t$.

Therefore the natural SS $(\nabla, *, E)$ is canonically extended to the whole orbit space $M$.

\section{The Coxeter-Shephard Frobenius structures}

4.1. The finite Coxeter groups and the Shephard groups. The duality groups include the finite Coxeter group 3 and the Shephard groups. For an irreducible finite complex reflection group $G$, the following conditions are equivalent (see [9, Theorem 6.121]):

(CS1) $G$ is a finite Coxeter group or a Shephard group.

$(\mathrm{CS} 2) \operatorname{Hess}\left(x^{n}\right): \operatorname{Der}_{\mathbb{C}[V]}^{G} \rightarrow \Omega_{\mathbb{C}[V]}^{G}$ is an isomorphism.

(CS3) $d_{\alpha}+d_{n+1-\alpha}=d_{1}+d_{n}(1 \leq \alpha \leq n)$.

In this section, $G$ is a finite Coxeter group or a Shephard group. Let $x^{1}, \ldots, x^{n}$ be a set of basic invariants for $G$.

From the classification, we can see that the strict inequalities

$$
d_{1}>d_{2}>\ldots>d_{n}
$$

hold for a finite Coxeter group or a Shephard group G. The condition (CS3) and eq. (4.1) together imply that

$$
d_{\alpha}+d_{\beta}>d_{1}+d_{n} \quad(\alpha+\beta<n+1), \quad d_{\alpha}+d_{\beta}<d_{1}+d_{n} \quad(\alpha+\beta>n+1) .
$$

Now let $h$ be a symmetric bilinear form on $T M^{\circ}$ corresponding to the map $\operatorname{Hess}\left(x^{n}\right)$, i.e.

$$
h(\cdot, \cdot)=\left\langle\operatorname{Hess}\left(x^{n}\right)(\cdot), \cdot\right\rangle
$$

The condition (CS2) implies that $h$ is a metric on $T M^{\circ}$. Let $\nabla^{c s}$ be the Levi-Civita connection for the metric $h$. In the local $u$-coordinates, $h$ is given by

$$
\tilde{H}_{i j}:=h\left(\frac{\partial}{\partial u^{i}}, \frac{\partial}{\partial u^{j}}\right)=\left\langle\operatorname{Hess}\left(x^{n}\right)\left(\frac{\partial}{\partial u^{i}}\right), \frac{\partial}{\partial u^{j}}\right\rangle=\frac{\partial^{2} x^{n}}{\partial u^{i} \partial u^{j}},
$$

where $\langle$,$\rangle is the canonical pairing. The Levi-Civita connection \nabla^{c s}$ is expressed as

$$
\boldsymbol{\nabla}_{\frac{\partial}{\partial u^{i}}}^{c s}\left(\frac{\partial}{\partial u^{j}}\right)=\sum_{k=1}^{n} \tilde{S}_{i j}^{k} \frac{\partial}{\partial u^{k}}, \quad \tilde{S}_{i j}^{k}=\frac{1}{2} \sum_{l=1}^{n} \tilde{H}^{k l} \frac{\partial^{3} x^{n}}{\partial u^{i} \partial u^{j} \partial u^{l}}
$$

\footnotetext{
${ }^{3}$ Among the duality groups, the finite Coxeter groups are characterized by the property that $d_{n}=2$.
} 
Here $\tilde{H}^{i j}(1 \leq i, j \leq n)$ denotes the $(i, j)$ entry of the inverse matrix $\tilde{H}^{-1}$ of $\tilde{H}=\left(\tilde{H}_{i j}\right)$. From this expression, we can immediately see that $\tilde{S}_{i j}^{k}=0$ holds if $d_{n}=2$. In other words, $\nabla^{c s}=\nabla^{V}$ if $G$ is a finite Coxeter group.

Example 4.1. For $G=\mathbb{Z}_{m}(m \geq 2), n=1, x^{1}=\left(u^{1}\right)^{m}, d_{1}=m$. Therefore

$$
\tilde{H}_{11}=m(m-1)\left(u^{1}\right)^{m-2}, \quad \tilde{S}_{11}^{1}=\frac{m-2}{2 u^{1}} .
$$

Example 4.2. For $G=G(m, 1, n)(m \geq 3, n \geq 2), x^{n}=\left(u^{1}\right)^{m}+\cdots+\left(u^{n}\right)^{m}$. Therefore

$$
\tilde{H}_{i j}=\left\{\begin{array}{ll}
m(m-1)\left(u^{i}\right)^{m-2} & (i=j) \\
0 & (i \neq j)
\end{array},\right.
$$

and

$$
\tilde{S}_{i j}^{k}=\left\{\begin{array}{ll}
\frac{m-2}{2 u^{i}} & (i=j=k) \\
0 & \text { (otherwise) }
\end{array} .\right.
$$

Lemma 4.3. (1) $\nabla^{c s}$ is flat.

(2) $\boldsymbol{\nabla}^{\text {cs }}$ and $E$ (given in (3.4)) satisfy (ASS3) with $r=\frac{d_{n}}{2 d_{1}}$ :

$$
\nabla_{X}^{c s} E=\frac{d_{n}}{2 d_{1}} X \quad\left(X \in \mathcal{T}_{M^{\circ}}\right)
$$

Proof. Let us set

$$
\left(\tilde{S}_{i}\right)^{k}{ }_{j}=\tilde{S}_{i j}^{k} \quad(1 \leq i \leq n)
$$

(1) The flatness is equivalent to

$$
\frac{\partial \tilde{S}_{j}}{\partial u^{i}}-\frac{\partial \tilde{S}_{i}}{\partial u^{j}}+\tilde{S}_{i} \tilde{S}_{j}-\tilde{S}_{j} \tilde{S}_{i} \stackrel{(4.5)}{=}-\left(\tilde{S}_{i} \tilde{S}_{j}-\tilde{S}_{j} \tilde{S}_{i}\right)=O
$$

If $G$ is a finite Coxeter group (i.e. if $\left.d_{n}=2\right)$, then $\tilde{S}_{i}=O(1 \leq i \leq n)$ by (4.5). In the cases $G=G_{3} \cong \mathbb{Z}_{m}(m \geq 1)$ and $G=G(m, 1, n)(m \geq 3, n \geq 2)$, it is easy to check that $\tilde{S}_{i j}^{k}$ 's obtained in Example 4.1 and Example 4.2 satisfy (4.6). For the remaining exceptional groups, $x^{n}$ can be found in [7, Chapter 6], [9, §B.3]. We checked that (4.6) holds using Mathematica.

(2) Recall that the derivation $E_{\text {deg }}$ in $(3.3)$ acts on a homogeneous polynomial $f \in \mathbb{C}[x]=$ 

$\mathbb{C}[u]^{G}$ by $E_{\operatorname{deg}}(f)=(\operatorname{deg} f) f($ see $(3.4))$. So we have

$$
\begin{aligned}
\boldsymbol{\nabla}_{\frac{\partial}{\partial u^{i}}}^{c s} E & =\frac{1}{d_{1}}\left(\frac{\partial}{\partial u^{i}}+\frac{1}{2} \sum_{j, k=1}^{n} u^{j} \tilde{S}_{i j}^{k} \frac{\partial}{\partial u^{k}}\right) \\
& \stackrel{(4.5)}{=} \frac{1}{d_{1}}\left(\frac{\partial}{\partial u^{i}}+\frac{1}{2} \sum_{j, k, l=1}^{n} u^{j} \frac{\partial \tilde{H}_{i l}}{\partial u^{j}} \cdot \tilde{H}^{k l} \frac{\partial}{\partial u^{k}}\right) \\
& =\frac{1}{d_{1}}\left(\frac{\partial}{\partial u^{i}}+\frac{d_{n}-2}{2} \sum_{k, l=1}^{n} \tilde{H}_{i l} \tilde{H}^{k l} \frac{\partial}{\partial u^{k}}\right) \\
& =\frac{1}{d_{1}}\left(\frac{\partial}{\partial u^{i}}+\frac{d_{n}-2}{2} \frac{\partial}{\partial u^{i}}\right)=\frac{d_{n}}{2 d_{1}} \frac{\partial}{\partial u^{i}} .
\end{aligned}
$$

Theorem 4.4. (1) The endomorphism

$$
T_{p} M^{\circ} \rightarrow T_{p} M^{\circ}, \quad X \mapsto \nabla_{X}^{c s} e
$$

of the tangent space $T_{p} M^{\circ}$ is invertible at every point $p \in M^{\circ}$. Therefore the following condition uniquely determines the multiplication $\diamond$ on $T M^{\circ}$ :

$$
\nabla_{X}^{c s} \nabla_{Y}^{c s} e-\nabla_{\nabla_{X}^{c s} Y}^{c s} e+\nabla_{X \diamond Y}^{c s} e=0 \quad\left(X, Y \in \mathcal{T}_{M^{\circ}}\right)
$$

(2) The multiplication $\diamond$ is associative and commutative and has the unit E.

(3) $\left(\boldsymbol{\nabla}^{c s}, \diamond, e\right)$ is an ASS with parameter $\frac{d_{n}}{2 d_{1}}$ on $M^{\circ}$.

(4) $(h, \diamond, e)$ is an almost Frobenius structure of charge $1-\frac{d_{n}}{d_{1}}$ on $M^{\circ}$.

The proof of Theorem 4.4 will be given in $\$ 7$.

Now let $\circledast$ be the multiplication dual to $\diamond$ and let $\nabla^{c s}$ be the connection dual to $\nabla^{c s}$ (see (2.1), (2.2)):

$$
e \diamond(X \circledast Y)=X \diamond Y, \quad \nabla_{X}^{c s} Y=\nabla_{X}^{c s} Y-\nabla_{X \circledast Y}^{c s} e .
$$

Notice that the converse relations (2.3) and (2.4) become

$$
E \circledast(X \diamond Y)=X \circledast Y, \quad \nabla_{X}^{c s} Y=\nabla_{X}^{c s} Y+\frac{d_{n}}{2 d_{1}} X \diamond Y-\nabla_{X \diamond Y} E .
$$

Then the multiplication $\circledast$ has $e$ as the unit. Moreover, by the almost duality, $\left(\nabla^{c s}, \circledast, E\right)$ is a $\mathrm{SS}$ on $M^{\circ}$. The following theorem says that this $\mathrm{SS}$ can be extended to $M$.

Theorem 4.5. The $S S\left(\nabla^{c s}, \circledast, E\right)$ is a polynomial $S S$ on $M$.

The proof will be given in $\$ 7.4$.

Let $\eta$ be the metric dual to $h$ (see (2.11) ):

$$
\eta(X, Y)=h(X, E \circledast Y) .
$$


Then $(\eta, \circledast, E)$ is a Frobenius structure on $M$ of charge $D=1-\frac{d_{n}}{d_{1}}$ which has $\left(\nabla^{c s}, \circledast, E\right)$ as the underlying Saito structure. In this article, we call $(\eta, \circledast, E)$ the Coxeter-Shephard (CS) Frobenius structure.

\section{Relationship Between the two Saito Structures}

Let $G$ be a finite Coxeter group or a Shephard group.

Recall that a finite Coxeter group or a Shephard group is a duality group. Therefore we have two ASS's for $G$. The one is the natural ASS $\left(\nabla^{V}, \star, e\right)$ with parameter $\frac{1}{d_{1}}$ explained in $₫ 3.2$ and the other is $\left(\nabla^{c s}, \diamond, e\right)$ with parameter $\frac{d_{n}}{2 d_{1}}$ explained in $₫ 4$. So it is natural to ask whether they are the same or not. It is clear that the parameters $\frac{d_{n}}{2 d_{1}}$ and $\frac{1}{d_{1}}$ agree if and only if $d_{n}=2$ i.e. $G$ is a finite Coxeter group. The connection $\nabla^{c s}$ agree with $\nabla^{V}$ agree if and only if $\left(\tilde{H}_{i j}\right)$ is a constant matrix, i.e. $d_{n}=2$. As for the multiplication, for any finite Coxeter group or any Shephard group $G$, we have the following

Theorem 5.1. The multiplication $\star$ and the multiplication $\diamond$ are the same.

The proof of Theorem 5.1 will be given in $\$ 7.1$

Corollary 5.2. The two $A S S^{\prime} s\left(\nabla^{V}, \star, e\right)$ and $\left(\nabla^{c s}, \diamond, e\right)$ for $G$ agree if and only if $d_{n}=2$, i.e. if and only if $G$ is a finite Coxeter group.

Similarly, we may ask whether the two dual Saito structures $(\nabla, *, E)$ and $\left(\nabla^{c s}, \circledast, E\right)$ are the same or not. Theorem 5.1 and the first equations of (3.7), (4.8) imply the following

Corollary 5.3. The multiplication $\circledast$ and the multiplication $*$ are the same.

As for the connections, the second equations of (3.7), (3.8), (4.8), (4.9) imply that $\nabla^{c s}=\nabla$ holds if and only if

$$
\nabla_{X}^{c s} Y=\nabla_{X}^{V} Y+\frac{d_{n}-2}{2 d_{1}} X \star Y \quad\left(X, Y \in \mathcal{T}_{M}\right)
$$

In the $u$-coordinates, the above relation can be expressed as

$$
\tilde{S}_{i j}^{k}=\frac{d_{n}-2}{2 d_{1}} \tilde{B}_{i j}^{k}
$$

Using the classification of the Shepard groups, we obtain the following

Theorem 5.4. (1) $\nabla=\nabla^{c s}$ holds if and only if $G$ is a finite Coxeter group or one of the following groups:

$$
G_{3} \cong \mathbb{Z}_{m}, \quad G_{4}, \quad G_{5}, \quad G_{8}, \quad G_{16}, \quad G_{20}, \quad G_{25}, \quad G_{32}
$$

(2) The Saito structures $(\nabla, *, E)$ and $\left(\nabla^{c s}, \circledast, E\right)$ for $G$ agree if and only if $G$ is a finite Coxeter group or one of the above groups. 
Proof. If $G$ is a finite Coxeter group, (5.1) is true since $\tilde{S}_{i j}^{k}=0$ and $d_{n}=2$. For $G=\mathbb{Z}_{m}$ $(m \geq 2)$, we can see (5.1) holds by comparing Examples 3.3 and 4.1 . For $G=G(m, 1, n)$ $(m \geq 3, n \geq 2)$, we see (5.1) does not hold by comparing Examples 3.4 and 4.2 . For the remaining exceptional Shephard groups, we checked whether (5.1) holds or not using Mathematicat and obtained the result.

Studying the condition $\nabla=\nabla^{c s}$ in the $x$-coordinates, we are led to the following

Theorem 5.5. The natural $S S(\nabla, *, E)$ admits a compatible Frobenius structure if and only if $\nabla=\nabla^{c s}$. Moreover, a compatible metric is a constant multiple of $\eta$ and the charge is $D=1-\frac{d_{n}}{d_{1}}$.

The proof will be given in 98 ,

\section{The MATRIX REPRESENTATION With RESPECT TO $\nabla$-FLAT COORDINATES}

Let $G$ be a finite Coxeter group or a Shephard group of rank $n$ and let $x^{1}, \ldots, x^{n}$ be a set of basic invariants for $G$. We take $e$ and $h$ as in (3.2) and (4.3). Denote by $(\nabla, *, E)$ the natural Saito structure for $G$ with the unit $e$. For the sake of convenience, we take a system of $\nabla$-flat coordinates $t=\left(t^{1}, \ldots, t^{n}\right)$ satisfying (i) and (ii) in Theorem 3.5. Here we choose the normalization $t^{1}=x^{1}+\mathbb{C}\left[x^{2}, \ldots, x^{n}\right], t^{n}=x^{n}$ so that $e$ and $h$ are unchanged, i.e.

$$
e=\frac{\partial}{\partial t^{1}}, \quad h(\cdot, \cdot)=\left\langle\operatorname{Hess}\left(t^{n}\right)(\cdot), \cdot\right\rangle
$$

Notice that $E_{\mathrm{deg}}$ and $E$ defined in (3.3) is also written as

$$
E_{\mathrm{deg}}=\sum_{\alpha=1}^{n} d_{\alpha} t^{\alpha} \frac{\partial}{\partial t^{\alpha}}, \quad E=\frac{1}{d_{1}} E_{\mathrm{deg}} .
$$

Below we write $\mathbb{C}\left[t^{1}, t^{2}, \ldots t^{n}\right]=\mathbb{C}[t], \mathbb{C}\left[t^{\prime}\right]=\mathbb{C}\left[t^{2}, \ldots, t^{n}\right]$ and

$$
\partial_{\alpha}=\frac{\partial}{\partial t^{\alpha}} \quad(1 \leq \alpha \leq n)
$$

The $\nabla$-flatness is expressed as $\nabla\left(\partial_{\alpha}\right)=0(1 \leq \alpha \leq n)$.

6.1. Matrix representations. In this subsection, we write down the conditions for the natural $\operatorname{SS}(\nabla, *, E)$ and the natural $\operatorname{ASS}\left(\nabla^{V}, \star, e\right)$ in the the matrix form with respect to the $\nabla$-flat coordinates $t$. (See also $[6, \S 5]$ ).

For two $n \times n$ matrices $A$ and $B,[A, B]:=A B-B A$. The identity matrix and the zero matrix are denoted $I$ and $O . M_{n}(\mathbb{C}[t])$ denote the space of $n \times n$ matrices whose entries are polynomials in $t$.

\footnotetext{
${ }^{4}$ Given a set of basic invariants, it is not difficult to compute $\tilde{S}_{i j}^{k}$ and $\tilde{B}_{i j}^{k}$ using Mathematica. See (3.6) and (4.5). Formulas for basic invariants can be found, e.g., in [7, 9]. For $G_{32}$, see [8].
} 
First, let us consider the conditions for the natural SS $(\nabla, *, E)$ for $G$. Denote by $C_{\alpha \beta}^{\gamma}$ $(1 \leq \alpha, \beta, \gamma \leq n)$ the structure constants of the multiplication $*$ :

$$
\partial_{\alpha} * \partial_{\beta}=\sum_{\gamma=1}^{n} C_{\alpha \beta}^{\gamma} \partial_{\gamma}, \quad\left(C_{\alpha}\right)_{\beta}^{\gamma}:=C_{\alpha \beta}^{\gamma} .
$$

The matrix $C_{\alpha}$ is the matrix representation of $\partial_{\alpha} *$ with respect to the basis $\left(\partial_{1}, \ldots, \partial_{n}\right)$. Since the multiplication $*$ is commutative, associative, and has $e=\partial_{1}$ as the unit, we have

$$
C_{\alpha \beta}^{\gamma}=C_{\beta \alpha}^{\gamma}, \quad\left[C_{\alpha}, C_{\beta}\right]=O, \quad C_{1}=I
$$

Denote by $U$ the representation matrix of $E *$ with respect to the basis $\partial_{1}, \ldots, \partial_{n}$ :

$$
E * \partial_{\beta}=\sum_{\gamma=1}^{n} U^{\gamma}{ }_{\beta} \partial_{\gamma}, \quad U=\sum_{\alpha=1}^{n} \frac{d_{\alpha}}{d_{1}} t^{\alpha} C_{\alpha} .
$$

Notice that the second equation of (6.2) implies

$$
\left[U, C_{\alpha}\right]=O \quad(1 \leq \alpha \leq n)
$$

Given that $\nabla\left(\partial_{\alpha}\right)=0$, the conditions (SS1), (SS2) are written as follows.

$$
\partial_{\alpha} C_{\beta}=\partial_{\beta} C_{\alpha}, \quad \partial_{\alpha} U=W C_{\alpha}-C_{\alpha} W+C_{\alpha} \quad(1 \leq \alpha, \beta \leq n),
$$

where

$$
W=\frac{1}{d_{1}} \operatorname{diag}\left(d_{1}, \ldots, d_{n}\right) .
$$

It is clear that $e=\partial_{1}$ and $E$ satisfy the conditions (SS3), (SS4).

Recall that the natural SS $(\nabla, *, E)$ is polynomial, i.e., $C_{\alpha \beta}^{\gamma} \in \mathbb{C}[t]$. By the first equation of (6.5) and by $C_{1}=I$, we have $\partial_{1} C_{\alpha}=\partial_{\alpha} C_{1}=O$. Therefore

$$
C_{\alpha} \in M_{n}\left(\mathbb{C}\left[t^{\prime}\right]\right), \quad U-t^{1} I \in M_{n}\left(\mathbb{C}\left[t^{\prime}\right]\right) .
$$

So $\operatorname{det} U \in \mathbb{C}[t]$ is a monic of degree $n$ as a polynomial in $t^{1}$. In fact, $\operatorname{det} U$ agrees with the discriminant polynomial of $G$ and

$$
M \backslash M^{\circ}=\{t \in M \mid \operatorname{det} U=0\} .
$$

Applying the derivation $E_{\mathrm{deg}}$ to $C_{\alpha \beta}^{\gamma}$, we obtain

$$
\begin{gathered}
\left(\operatorname{deg} C_{\alpha \beta}^{\gamma}\right) C_{\alpha \beta}^{\gamma} \stackrel{\stackrel{(3.4)}{=}}{=} E_{\operatorname{deg}} C_{\alpha \beta}^{\gamma}=\sum_{\mu=1}^{n} d_{\mu} t^{\mu} \partial_{\mu} C_{\alpha \beta}^{\gamma} \stackrel{(6.5)}{=} \sum_{\mu=1}^{n} d_{\mu} t^{\mu} \partial_{\alpha} C_{\mu \beta}^{\gamma} \\
=d_{1} \partial_{\alpha} U-d_{\alpha} C_{\alpha \beta}^{\gamma} \stackrel{(6.5)}{=}\left(d_{1}+d_{\gamma}-d_{\alpha}-d_{\beta}\right) C_{\alpha \beta}^{\gamma} .
\end{gathered}
$$

Therefore

$$
\operatorname{deg} C_{\alpha \beta}^{\gamma}=d_{1}+d_{\gamma}-d_{\alpha}-d_{\beta}, \quad \operatorname{deg} U_{\beta}^{\gamma}=d_{1}+d_{\gamma}-d_{\beta} .
$$


Next let us consider the ASS $\left(\nabla^{V}, \star, e\right)$ with parameter $r=\frac{1}{d_{1}}$ which is dual to $(\nabla, * E)$. Denote by $B_{\alpha \beta}^{\gamma}(1 \leq \alpha, \beta, \gamma \leq 1)$ the structure constants of the multiplication $\star$ :

$$
\partial_{\alpha} \star \partial_{\beta}=\sum_{\gamma=1}^{n} B_{\alpha \beta}^{\gamma} \partial_{\gamma}, \quad\left(B_{\alpha}\right)^{\gamma}{ }_{\beta}=B_{\alpha \beta}^{\gamma} .
$$

Substituting $X=\partial_{\alpha}$ and $Y=\partial_{\beta}$ into the first equation of (3.8), we have

$$
\sum_{\delta=1}^{n} U^{\gamma}{ }_{\delta} B_{\alpha \beta}^{\delta}=C_{\alpha \beta}^{\gamma}
$$

or $U B_{\alpha}=C_{\alpha}$. Thus,

$$
B_{\alpha}=U^{-1} C_{\alpha} \stackrel{\sqrt[6.4]{=}}{=} C_{\alpha} U^{-1}
$$

(Therefore the entries of $B_{\alpha}$ are homogeneous rational functions in $t$ with the denominator $\operatorname{det} U . B_{\alpha}$ is only defined on $M^{\circ}$.)

Eqs. (6.2) and (6.4) imply

$$
B_{\alpha \beta}^{\gamma}=B_{\beta \alpha}^{\gamma}, \quad\left[B_{\alpha}, C_{\beta}\right]=O, \quad\left[B_{\alpha}, B_{\beta}\right]=O, \quad\left[B_{\alpha}, U\right]=O
$$

Eq. (6.5) together with (6.9) implies that

$$
\begin{aligned}
\partial_{\alpha} B_{\beta} & =\partial_{\alpha}\left(C_{\beta} U^{-1}\right)=\left(\partial_{\alpha} C_{\beta}\right) U^{-1}-C_{\beta} U^{-1}\left(\partial_{\alpha} U\right) U^{-1} \\
& =\left(\partial_{\alpha} C_{\beta}\right) U^{-1}-B_{\beta} W B_{\alpha}+U^{-1} C_{\beta} C_{\alpha} W U^{-1}-B_{\beta} B_{\alpha} .
\end{aligned}
$$

Especially, if $\alpha=1, C_{1}=I$ and $\partial_{1} C_{\beta}=\partial_{\beta} I=O$. So we have

$$
\partial_{1} B_{\beta}=-B_{\beta} U^{-1}
$$

Now let $\Omega_{\alpha}$ be the connection matrix of $\nabla^{V}$ :

$$
\nabla_{\partial_{\alpha}}^{V}\left(\partial_{\beta}\right)=\sum_{\gamma=1}^{n} \Omega_{\alpha \beta}^{\gamma} \partial_{\gamma}, \quad\left(\Omega_{\alpha}\right)_{\beta}^{\gamma}=\Omega_{\alpha \beta}^{\gamma} .
$$

Substituting $X=\partial_{\alpha}, Y=\partial_{\beta}$ into the second equation of (3.8), we obtain

$$
\Omega_{\alpha \beta}^{\gamma}=\frac{1-d_{\gamma}}{d_{1}} B_{\alpha \beta}^{\gamma} .
$$

Moreover, (3.1) implies that

$$
\Omega_{\alpha \beta}^{\gamma}=-\sum_{i, j=1}^{n} \frac{\partial u^{i}}{\partial t^{\alpha}} \frac{\partial u^{j}}{\partial t^{\beta}} \frac{\partial^{2} t^{\gamma}}{\partial u^{i} \partial u^{j}} .
$$


6.2. Representation matrix of the metric $h$. In the $t$-coordinates, the metric $h$ is given by

$$
H_{\alpha \beta}:=h\left(\partial_{\alpha}, \partial_{\beta}\right) \stackrel{(4.4)}{=} \sum_{i, j=1}^{n} \frac{\partial u^{i}}{\partial t^{\alpha}} \frac{\partial u^{j}}{\partial t^{\beta}} \frac{\partial^{2} t^{n}}{\partial u^{i} \partial u^{j}}, \quad H=\left(H_{\alpha \beta}\right) .
$$

Comparing this with (6.13), we have

$$
H_{\alpha \beta}=-\Omega_{\alpha \beta}^{n}
$$

Therefore from (6.12), we obtain a key relation

$$
H_{\alpha \beta}=-\Omega_{\alpha \beta}^{n}=\frac{d_{n}-1}{d_{1}} B_{\alpha \beta}^{n} .
$$

6.3. Representation matrix of $\operatorname{Hess}\left(t^{n}\right)$. Define vector fields $X_{\beta}(1 \leq \beta \leq n)$ on $M$ by

$$
X_{\beta}=E * \partial_{\beta}=\sum_{\gamma=1}^{n} U^{\gamma}{ }_{\beta} \partial_{\gamma}
$$

They are $G$-invariant vector fields on $V$ and form a basis of the $\mathbb{C}[V]^{G}$-module $\operatorname{Der}_{\mathbb{C}[V]}^{G}$ of $G$-invariant vector fields on $V[6, \S 7.3]$. Take $d t^{1}, \ldots, d t^{n}$ as a basis of the $\mathbb{C}[V]^{G}$-module $\Omega_{\mathbb{C}[V]}^{G}$ of $G$-invariant 1-forms on $V$. Let $A=\left(A_{\alpha \beta}\right)$ be the representation matrix of Hess $\left(t^{n}\right)$ with respect to them:

$$
\operatorname{Hess}\left(t^{n}\right)\left(X_{\beta}\right)=\sum_{\alpha=1}^{n} A_{\alpha \beta} d t^{\alpha}
$$

Notice that

$$
A_{\alpha \beta} \in \mathbb{C}[t], \quad \operatorname{det} A \neq 0,
$$

since $\operatorname{Hess}\left(t^{n}\right)$ is the isomorphism (see (CS2) in §4).

Lemma 6.1. $A=H U$ and

$$
A_{\alpha \beta}=\frac{d_{n}-1}{d_{1}} C_{\alpha \beta}^{n} \quad \in \mathbb{C}\left[t^{\prime}\right]
$$

Proof. First we show $A=H U$. By definition of $A_{\alpha \beta}$,

$$
\begin{aligned}
A_{\alpha \beta}= & \left\langle\operatorname{Hess}\left(t^{n}\right)\left(X_{\beta}\right), \partial_{\alpha}\right\rangle \stackrel{\sqrt{6.15}}{=} \sum_{\gamma=1}^{n} U_{\beta}^{\gamma}\left\langle\operatorname{Hess}\left(t^{n}\right)\left(\partial_{\gamma}\right), \partial_{\alpha}\right\rangle \\
& =\sum_{\gamma=1}^{n} U^{\gamma}{ }_{\beta} h\left(\partial_{\gamma}, \partial_{\alpha}\right)=\sum_{\gamma=1}^{n} U^{\gamma}{ }_{\beta} H_{\gamma \alpha}=\sum_{\gamma=1}^{n} U^{\gamma}{ }_{\beta} H_{\alpha \gamma} \\
& =(H U)_{\alpha \beta} .
\end{aligned}
$$

To show the second statement, recall (6.14).

$$
A_{\alpha \beta}=\sum_{\gamma=1}^{n} H_{\alpha \gamma} U_{\beta}^{\gamma} \stackrel{(6.14)}{=} \frac{d_{n}-1}{d_{1}}\left(B_{\alpha} U\right)_{\beta}^{n} \stackrel{(6.8)}{=} \frac{d_{n}-1}{d_{1}} C_{\alpha \beta}^{n} .
$$

Then $A_{\alpha \beta} \in \mathbb{C}\left[t^{\prime}\right]$ follows from (6.6). 
Lemma 6.2. (1) $\operatorname{deg} A_{\alpha \beta}=d_{1}+d_{n}-d_{\alpha}-d_{\beta}$ and

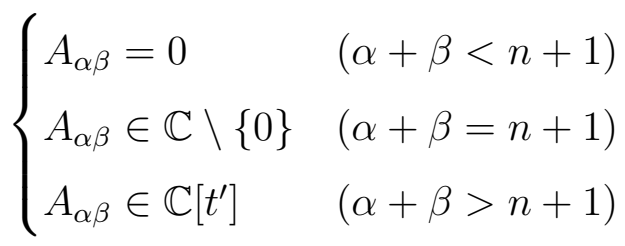

(2) $A^{-1} \in M_{n}\left(\mathbb{C}\left[t^{\prime}\right]\right)$.

(3) $A^{-1} W A \in M_{n}\left(\mathbb{C}\left[t^{\prime}\right]\right)$ is upper triangular and its diagonal entries are

$$
\left(A^{-1} W A\right)_{\mu}^{\mu}=\frac{d_{n+1-\mu}}{d_{1}}=\frac{d_{1}+d_{n}-d_{\mu}}{d_{1}} .
$$

(4) $A^{-1} \partial_{\alpha} A \in M_{n}\left(\mathbb{C}\left[t^{\prime}\right]\right)$ is strictly upper triangular.

Proof. (1) By Lemma 6.1 and (6.7), the degree of $A_{\alpha \beta}$ is

$$
\operatorname{deg} A_{\alpha \beta}=\operatorname{deg} C_{\alpha \beta}^{n}=d_{1}+d_{n}-d_{\alpha}-d_{\beta} .
$$

Recall that $d_{\alpha}+d_{\beta}>d_{1}+d_{n}$ holds if $\alpha+\beta<n+1$ (see (4.2)). Therefore if $\alpha+\beta<n+1$, $\operatorname{deg} A_{\alpha \beta}<0$, hence $A_{\alpha \beta}=0$. Recall also that $d_{\alpha}+d_{\beta}=d_{1}+d_{n}$ if $\alpha+\beta=n+1$ (see (CS3) in (44). Therefore $A_{\alpha \beta}$ is a constant if $\alpha+\beta=n+1$. Then we have

$$
\operatorname{det} A=(-1)^{\frac{n(n-1)}{2}} \prod_{\alpha=1}^{n} A_{\alpha, n+1-\alpha} .
$$

Since $\operatorname{det} A \neq 0, A_{\alpha, n+1-\alpha} \neq 0$.

(2) Let us put

$$
T=\left(\begin{array}{lll} 
& & 1 \\
& & \\
1 & &
\end{array}\right) .
$$

Then $T A$ is the matrix obtained by exchanging the $i$-th row and the $(n+1-i)$-th row $(1 \leq i \leq n)$ of $A$. So $T A \in M_{n}\left(\mathbb{C}\left[t^{\prime}\right]\right)$ is upper triangular and its diagonal entries are nonzero constants $A_{n 1}, \ldots, A_{1 n}$. Therefore $T A$ is invertible, $(T A)^{-1}=A^{-1} T \in M_{n}\left(\mathbb{C}\left[t^{\prime}\right]\right)$ is upper triangular and its diagonal entries are nonzero constants. $A^{-1}$ is obtained from $A^{-1} T$ by exchanging the $j$-th column and the $(n+1-j)$-th column $(1 \leq j \leq n)$. Therefore $A^{-1} \in M_{n}\left(\mathbb{C}\left[t^{\prime}\right]\right)$.

(3) and (4) immediately follow from $A^{-1} W A=(T A)^{-1}(T W T)(T A)$ and $A^{-1} \partial_{\alpha} A=$ $(T A)^{-1} \partial_{\alpha}(T A)$.

Lemma 6.3. $A={ }^{t} A$ and

$$
A C_{\alpha}={ }^{t} C_{\alpha} A, \quad A B_{\alpha}={ }^{t} B_{\alpha} A \quad(1 \leq \alpha \leq n), \quad A U={ }^{t} U A
$$


Proof. By Lemma 6.1, we have

$$
A_{\mu \nu}=\frac{d_{n}-1}{d_{1}} C_{\mu \nu}^{n} \stackrel{(6.2)}{=} \frac{d_{n}-1}{d_{1}} C_{\nu \mu}^{n}=A_{\nu \mu} .
$$

We also have

$$
\left(A C_{\alpha}\right)_{\mu \nu}=\frac{d_{n}-1}{d_{1}}\left(C_{\mu} C_{\alpha}\right)_{\nu}^{n} \stackrel{(6.2)}{=} \frac{d_{n}-1}{d_{1}}\left(C_{\alpha} C_{\mu}\right)_{\nu}^{n}=\frac{d_{n}-1}{d_{1}} \sum_{\lambda=1}^{n} C_{\alpha \lambda}^{n} C_{\mu \nu}^{\lambda},
$$

and

$$
\left(A B_{\alpha}\right)_{\mu \nu}=\frac{d_{n}-1}{d_{1}}\left(C_{\mu} B_{\alpha}\right)_{\nu} \stackrel{(6.9)}{=} \frac{d_{n}-1}{d_{1}}\left(B_{\alpha} C_{\mu}\right)^{n}{ }_{\nu}=\frac{d_{n}-1}{d_{1}} \sum_{\lambda=1}^{n} B_{\alpha \lambda}^{n} C_{\mu \nu}^{\lambda} .
$$

In all of the above equations, the RHS's are symmetric with respect to the exchange of $\mu$ and $\nu$. So $A, A C_{\alpha}$ and $A B_{\alpha}$ are symmetric matrices. Therefore $A={ }^{t} A, A C_{\alpha}={ }^{t}\left(A C_{\alpha}\right)=$ ${ }^{t} C_{\alpha} A$ and $A B_{\alpha}={ }^{t}\left(A B_{\alpha}\right)={ }^{t} B_{\alpha} A$. The remaining equation $A U={ }^{t} U A$ easily follows from $A C_{\alpha}={ }^{t} C_{\alpha} A$ and (6.3).

6.4. Levi-Civita connections for Shephard groups. In the $t$-coordinates, the LeviCivita connection $\nabla^{c s}$ of the metric $h$ is expressed as

$$
\nabla_{\partial_{\alpha}}^{c s}\left(\partial_{\beta}\right)=\sum_{\gamma=1}^{n} S_{\alpha \beta}^{\gamma} \partial_{\gamma}, \quad S_{\alpha \beta}^{\gamma}=\frac{1}{2} \sum_{\delta=1}^{n} H^{\gamma \delta}\left(\partial_{\alpha} H_{\delta \beta}+\partial_{\beta} H_{\delta \alpha}-\partial_{\delta} H_{\alpha \beta}\right) .
$$

Here $H=\left(H_{\alpha \beta}\right)$ is the representation matrix of the metric $h$ defined in $\$ 6.2$ and $H^{\alpha \beta}$ $(1 \leq \alpha, \beta \leq n)$ denotes the $(\alpha, \beta)$ entry of the inverse matrix $H^{-1}$. We put

$$
\left(S_{\alpha}\right)_{\beta}^{\gamma}=S_{\alpha \beta}^{\gamma}
$$

\section{Lemma 6.4.}

$$
2 S_{\alpha}=A^{-1} \partial_{\alpha} A+\left(-I-W+A^{-1} W A\right) B_{\alpha}
$$

Proof. With (6.14) and (6.10), we have

$$
\begin{aligned}
\partial_{\alpha} H_{\delta \beta} & =\frac{d_{n}-1}{d_{1}} \partial_{\alpha} B_{\delta \beta}^{n} \\
& =\frac{d_{n}-1}{d_{1}}\left(\left(\partial_{\alpha} C_{\delta}\right) U^{-1}-B_{\delta} W B_{\alpha}+U^{-1} C_{\delta} C_{\alpha} W U^{-1}-B_{\delta} B_{\alpha}\right)_{\beta}^{n} .
\end{aligned}
$$

Therefore

$$
\begin{aligned}
\partial_{\alpha} H_{\delta \beta}-\partial_{\delta} H_{\alpha \beta} & \stackrel{(6.2)(6.9)}{=} \frac{d_{n}-1}{d_{1}}\left(B_{\alpha} W B_{\delta}-B_{\delta} W B_{\alpha}\right)_{\beta}^{n} \\
& \stackrel{(6.14)}{=}\left(H W B_{\delta}\right)_{\alpha \beta}-\left(H W B_{\alpha}\right)_{\delta \beta} \\
& \stackrel{(6.9)}{=}\left(H W B_{\beta}\right)_{\alpha \delta}-\left(H W B_{\alpha}\right)_{\delta \beta} .
\end{aligned}
$$


On the other hand, by (6.14) and Lemma 6.1,

$$
\begin{aligned}
\partial_{\beta} H_{\delta \alpha} & =\partial_{\beta} H_{\alpha \delta}=\frac{d_{n}-1}{d_{1}} \partial_{\beta} B_{\alpha \delta}^{n} \\
& =\frac{d_{n}-1}{d_{1}}\left(\partial_{\beta} C_{\alpha} U^{-1}-B_{\alpha} W B_{\beta}+U^{-1} C_{\alpha} C_{\beta} W U^{-1}-B_{\alpha} B_{\beta}\right)^{n} \\
& =\frac{d_{n}-1}{d_{1}}\left(\partial_{\alpha} C_{\beta} U^{-1}-B_{\alpha} W B_{\beta}+C_{\beta} B_{\alpha} W U^{-1}-C_{\beta} B_{\alpha} U^{-1}\right)^{n}{ }_{\delta} \\
& =\left(\left(\partial_{\alpha} A\right) U^{-1}\right)_{\beta \delta}-\left(H W B_{\beta}\right)_{\alpha \delta}+\left(A B_{\alpha} W U^{-1}\right)_{\beta \delta}-\left(A B_{\alpha} U^{-1}\right)_{\beta \delta}
\end{aligned}
$$

Adding these two equations, we obtain

$$
\partial_{\alpha} H_{\delta \beta}+\partial_{\beta} H_{\delta \alpha}-\partial_{\delta} H_{\alpha \beta}=\left(\left(\partial_{\alpha} A+A B_{\alpha}(W-I)\right) U^{-1}\right)_{\beta \delta}-\left(H W B_{\alpha}\right)_{\delta \beta} .
$$

Therefore

$$
\begin{aligned}
2 S_{\alpha \beta}^{\gamma} & =\sum_{\delta=1}^{n} H^{\gamma \delta}\left(\left(\partial_{\alpha} A+A B_{\alpha}(W-I)\right) U^{-1}\right)_{\beta \delta}-\sum_{\delta=1}^{n} H^{\gamma \delta}\left(H W B_{\alpha}\right)_{\delta \beta} \\
& =\left(\left(\partial_{\alpha} A+A B_{\alpha}(W-I)\right) U^{-1} H^{-1}\right)_{\beta}^{\gamma}-\left(W B_{\alpha}\right)_{\beta}^{\gamma} \\
& =\left(\left(\partial_{\alpha} A+A B_{\alpha}(W-I)\right) A^{-1}\right)_{\beta}^{\gamma}-\left(W B_{\alpha}\right)_{\beta}^{\gamma} .
\end{aligned}
$$

Using Lemma 6.3, we see that the matrix in the first term is the transpose of

$$
A^{-1} \partial_{\alpha} A+A^{-1}(W-I) A B_{\alpha} .
$$

Therefore

$$
2 S_{\alpha \beta}^{\gamma}=\left(A^{-1} \partial_{\alpha} A+\left(-I-W+A^{-1} W A\right) B_{\alpha}\right)_{\beta}^{\gamma}
$$

\section{Proofs of Theorem 4.4, Theorem 4.5 and Theorem 5.1}

\subsection{Proofs of Theorem 4.4-(1)(2) and Theorem 5.1. First we show that}

$$
\operatorname{det} S_{1} \neq 0
$$

holds on $M^{\circ}$. Substituting $\partial_{1} A=O$ and $B_{1}=U^{-1}$ into Lemma 6.4, we have

$$
2 S_{1}=\left(-I-W+A^{-1} W A\right) U^{-1} .
$$

By Lemma 6.2 (3), $-I-W+A^{-1} W A$ is upper triangular and

$$
\left(-I-W+A^{-1} W A\right)^{\mu}{ }_{\mu}=-1-\frac{d_{\mu}}{d_{1}}+\frac{d_{1}+d_{n}-d_{\mu}}{d_{1}}=\frac{-2 d_{\mu}+d_{n}}{d_{1}} \stackrel{\text { (4.1) }}{<} 0 .
$$

This implies $\operatorname{det}\left(-I-W+A^{-1} W A\right) \neq 0$. Since $\operatorname{det} U \neq 0$ on $M^{\circ}$, $\operatorname{det} S_{1} \neq 0$ on $M^{\circ}$. The representation matrix of the map $X \rightarrow \nabla_{X}^{c s} e$ is given by $S_{1}$. Therefore $\operatorname{det} S_{1} \neq 0$ implies that this map is invertible. This proves Theorem 4.4-(1). Thus (4.7) determines the multiplication $\diamond$. 
Next we prove Theorem 5.1. Let $B_{\alpha}^{c s}(1 \leq \alpha \leq n)$ denote the representation matrix of $\partial_{\alpha} \diamond$. To show that the multiplication $\diamond$ agrees with $\star$, it is enough to show that $B_{\alpha}^{c s}=B_{\alpha}$. Notice that (4.7) is written as follows.

$$
O=\partial_{\alpha} S_{1}+S_{\alpha} S_{1}-S_{1} S_{\alpha}+S_{1} B_{\alpha}^{c s}=\partial_{1} S_{\alpha}+S_{1} B_{\alpha}^{c s}
$$

In the last line, we used the flatness of $\nabla^{c s}$. Therefore

$$
B_{\alpha}^{c s}=-S_{1}^{-1} \partial_{1} S_{\alpha}
$$

Since $A$ is independent of $t^{1}$ (Lemma 6.2), we have

$$
2 \partial_{1} S_{\alpha}=\left(-I-W+A^{-1} W A\right) \partial_{1} B_{\alpha} \stackrel{\sqrt[6.11]{=}}{=}-\left(-I-W+A^{-1} W A\right) U^{-1} B_{\alpha} .
$$

Substituting this equation and (7.1) into (7.2), we obtain $B_{\alpha}^{c s}=B_{\alpha}$.

Theorem 4.4-(2) immediately follows from Theorem 5.1 .

7.2. Proof of Theorem 4.4 (3). We show that $\left(\nabla^{c s}, \diamond=\star, E\right)$ satisfies (ASS1)(ASS4). We already showed that $\boldsymbol{\nabla}^{c s}$ and $E$ satisfy (ASS3) in Lemma 4.3. It is clear that $\diamond$ satisfies (ASS2) since $\star=\diamond$ satisfies (ASS2). It is also clear that (ASS4) holds since the multiplication $\diamond$ is made from $\boldsymbol{\nabla}^{c s}$ and $e$ by the condition (ASS4). So we only have to check the condition (ASS1).

In the matrix representation, (ASS1) is equivalent to

$$
\partial_{\alpha} B_{\beta}+\left[S_{\alpha}, B_{\beta}\right]=\partial_{\beta} B_{\alpha}+\left[S_{\beta}, B_{\alpha}\right]
$$

Using Lemma 6.4, this is equivalent to

$$
\begin{aligned}
2\left(\partial_{\alpha} B_{\beta}-\partial_{\beta} B_{\alpha}\right) & =-\left[A^{-1} \partial_{\alpha} A, B_{\beta}\right]+\left[A^{-1} \partial_{\beta} A, B_{\alpha}\right] \\
& +B_{\beta}\left(-W+A^{-1} W A\right) B_{\alpha}-B_{\alpha}\left(-W+A^{-1} W A\right) B_{\beta} .
\end{aligned}
$$

To show (7.3), let us compute $\partial_{\alpha} B_{\beta}-\partial_{\beta} B_{\alpha}$ in two ways. By (6.10), we have

$$
\partial_{\alpha} B_{\beta}-\partial_{\beta} B_{\alpha}=-B_{\beta} W B_{\alpha}+B_{\alpha} W B_{\beta}
$$

On the other hand, using $B_{\alpha}=A^{-1}\left({ }^{t} B_{\alpha}\right) A$ (Lemma 6.3), we have

$$
\begin{aligned}
& \partial_{\alpha} B_{\beta}-\partial_{\beta} B_{\alpha}=\partial_{\alpha}\left(A^{-1 t} B_{\beta} A\right)-\partial_{\beta}\left(A^{-1 t} B_{\alpha} A\right) \\
& =-A^{-1} \partial_{\alpha} A \underbrace{A^{-1 t} B_{\beta} A}_{=B_{\beta}}+A^{-1} \partial_{\alpha}{ }^{t} B_{\beta} A+\underbrace{A^{-1} B_{\beta}}_{=B_{\beta} A^{-1}} \partial_{\alpha} A-(\alpha \leftrightarrow \beta) \\
& =-\left[A^{-1} \partial_{\alpha} A, B_{\beta}\right]+\left[A^{-1} \partial_{\beta} A, B_{\alpha}\right]+A^{-1 t}\left(\partial_{\alpha} B_{\beta}-\partial_{\beta} B_{\alpha}\right) A^{-1} \\
& =-\left[A^{-1} \partial_{\alpha} A, B_{\beta}\right]+\left[A^{-1} \partial_{\beta} A, B_{\alpha}\right]-B_{\alpha} A^{-1} W A B_{\beta}+B_{\beta} A^{-1} W A B_{\alpha} .
\end{aligned}
$$

Adding these two equations, we obtain (7.3). 
7.3. Proof of Theorem 4.4 (4). To show that $(h, \star, e)$ is an almost Frobenius structure, we have to check (2.8), (2.9), (2.10) with $\nabla, g$ replaced by $\nabla^{c s}, h$. Eq. (2.8) trivially holds since $\nabla^{c s}$ is the Levi-Civita connection of $h$. In the matrix form, (2.9) and (2.10) are equivalent to the followings.

$$
\sum_{\lambda=1}^{n} B_{\alpha \beta}^{\lambda} H_{\lambda \gamma}=\sum_{\lambda=1}^{n} B_{\beta \gamma}^{\lambda} H_{\alpha \lambda}, \quad \partial_{1} H+H B_{1}=O .
$$

But these immediately follows from (6.14), (6.9) and (6.11).

7.4. Proof of Theorem 4.5. The proof is almost the same as the proof of 6 , Theorem 7.5-(3)].

Consider the Saito structure $\left(\nabla^{c s}, \circledast, e\right)$ dual to $\left(\nabla^{c s}, \diamond=\star, E\right)$. Comparing the first equations of (3.7) and (4.8), we see that the multiplication $\circledast$ agrees with the multiplication $*$ of the natural Saito structure for $G$. To show that $\left(\nabla^{c s}, \circledast=*, e\right)$ is a polynomial Saito structure, we will find a set of basic invariants $s=\left(s^{1}, \ldots, s^{n}\right)$ satisfying the following (i) and (ii):

(i) $s=\left(s^{1}, s^{2}, \ldots, s^{n}\right)$ is a system of $\nabla^{c s}$-flat coordinates.

(ii) The structure constants of the multiplication $\circledast=*$ with respect to the basis $\frac{\partial}{\partial s^{1}}, \ldots, \frac{\partial}{\partial s^{n}}$ are polynomials in $s$.

Since $\circledast=*$, the representation matrix of $\partial_{\alpha} \circledast$ is $C_{\alpha}$. Let

$$
\nabla_{\partial_{\alpha}}^{c s}\left(\partial_{\beta}\right)=\sum_{\gamma=1}^{n} \Upsilon_{\alpha \beta}^{\gamma}, \quad\left(\Upsilon_{\alpha}\right)_{\beta}^{\gamma}=\Upsilon_{\alpha \beta}^{\gamma}
$$

Then by the second relation of (4.8) and Lemma 6.4,

$$
\Upsilon_{\alpha}=S_{\alpha}-S_{1} C_{\alpha}=\frac{1}{2} A^{-1} \partial_{\alpha} A \quad(1 \leq \alpha \leq n) .
$$

Notice that Lemma 6.2 implies that $\Upsilon_{\alpha}$ is strictly upper triangular and that $\operatorname{deg} \Upsilon_{\alpha \beta}^{\gamma}=$ $d_{\gamma}-d_{\alpha}-d_{\beta}$. Moreover, the flatness 5 of $\nabla^{c s}$ implies that

$$
\partial_{\alpha} \Upsilon_{\beta}-\partial_{\beta} \Upsilon_{\alpha}+\left[\Upsilon_{\alpha}, \Upsilon_{\beta}\right]=O
$$

Lemma 7.1. There exists a unique upper unitriangular matrix $X \in M_{n}\left(\mathbb{C}\left[t^{\prime}\right]\right)$ with homogeneous entries satisfying

$$
\partial_{\alpha} X+\Upsilon_{\alpha} X=O \quad(1 \leq \alpha \leq n)
$$

Moreover $\operatorname{deg} X_{\beta}^{\gamma}=d_{\gamma}-d_{\beta}$.

\footnotetext{
${ }^{5}$ The connection $\nabla^{c s}$ is flat since it is constructed from $\nabla^{c s}$ by (4.8). See [6, Proposition 3.7].
} 
Proof. Let us set

$$
X_{\gamma}^{\gamma}=1 \quad(1 \leq \gamma \leq n), \quad X_{\beta}^{\gamma}=0 \quad(1 \leq \beta<\gamma \leq n)
$$

We will solve the equation (17.6). Component-wise, it is written as

$$
\partial_{\alpha} X_{\beta}^{\gamma}=-\sum_{\delta=1}^{n} \Upsilon_{\alpha \delta}^{\gamma} X_{\beta}^{\delta} \quad(1 \leq \alpha \leq n) .
$$

Notice that the sum in the RHS is taken for $\gamma<\delta \leq \beta$ since $\Upsilon_{\alpha \delta}^{\gamma}=0$ if $\gamma \geq \delta$ and $X_{\beta}^{\delta}=0$ if $\delta>\beta$.

Now let us fix $1 \leq \beta \leq n$. If $\gamma \geq \beta$, the RHS of (7.7) is zero because $\delta$ satisfying $\gamma<\delta \leq \beta$ does not exist. The LHS is also zero since $X_{\beta}^{\gamma}=0$ or 1. So (17.7) holds.

Consider the case $\gamma=\beta-1$. The system of partial differential equations (7.7) for $X^{\beta-1}$ becomes

$$
\partial_{\alpha} X_{\beta}^{\beta-1}=-\Upsilon_{\alpha \beta}^{\beta-1} \quad(1 \leq \alpha \leq n)
$$

The homogeneous polynomial solution $X^{\beta-1}{ }_{\beta} \in \mathbb{C}[t]$ uniquely exists due to (17.5). Its degree is

$$
\operatorname{deg} X_{\beta}^{\beta-1}=\operatorname{deg} \Upsilon_{\alpha \beta}^{\beta-1}+d_{\alpha}=\left(d_{\beta-1}-d_{\alpha}-d_{\beta}\right)+d_{\alpha}=d_{\beta-1}-d_{\beta}
$$

For $\gamma=\beta-2$, (7.7) becomes

$$
\partial_{\alpha} X_{\beta}^{\beta-2}=-\Upsilon_{\alpha \beta}^{\beta-2}-\Upsilon_{\alpha, \beta-1}^{\beta-2} X_{\beta}^{\beta-1} \quad(1 \leq \alpha \leq n)
$$

The homogeneous polynomial solution $X^{\beta-2}{ }_{\beta} \in \mathbb{C}[t]$ uniquely exists due to (7.5) and its degree is $d_{\beta-2}-d_{\beta}$. For $\gamma=\beta-3, \ldots, 2,1$, the similar argument shows the existence of homogeneous polynomial solution $X^{\gamma}{ }_{\beta} \in \mathbb{C}[t]$ of degree $d_{\gamma}-d_{\beta}$.

Since $\operatorname{deg} X_{\beta}^{\gamma}<d_{\gamma} \leq d_{1}, X_{\beta}^{\gamma}$ is independent of $t^{1}$, i.e. $X_{\beta}^{\gamma} \in \mathbb{C}\left[t^{\prime}\right]$.

Now let $X \in M_{n}\left(\mathbb{C}\left[t^{\prime}\right]\right)$ be the matrix in Lemma 7.1. Since $X \in M_{n}\left(\mathbb{C}\left[t^{\prime}\right]\right)$ is upper unitriangular, $X$ is invertible and $X^{-1} \in M_{n}\left(\mathbb{C}\left[t^{\prime}\right]\right)$ is also upper unitriangular. Moreover $\operatorname{deg}\left(X^{-1}\right)_{\beta}^{\gamma}=d_{\gamma}-d_{\beta}$. Then we can find homogeneous polynomials $s^{1}, \ldots, s^{n} \in \mathbb{C}[t]$ satisfying

$$
d s^{\alpha}=\sum_{\beta=1}^{n}\left(X^{-1}\right)^{\alpha}{ }_{\beta} d t^{\beta}, \quad \operatorname{deg} s^{\alpha}=d_{\alpha} \quad(1 \leq \alpha \leq n) .
$$

By degree consideration, $s^{1}, \ldots, s^{n}$ are of the following forms:

$$
s^{n}=t^{n}, \quad s^{n-1}=t^{n-1}+F_{n-1}\left(t^{n}\right), \quad \ldots, \quad s^{1}=t^{1}+F_{1}\left(t^{2}, \ldots, t^{n}\right) .
$$

We can solve these equations for $t$ and express $t^{1}, \ldots, t^{n}$ as polynomials in $s^{1}, \ldots, s^{n}$. Therefore $s=\left(s^{1}, \ldots, s^{n}\right)$ is a set of basic invariants. We obtain $\mathbb{C}[s]=\mathbb{C}[t]$ and $\mathbb{C}\left[s^{\prime}\right]=$ $\mathbb{C}\left[t^{\prime}\right]$. 
Next let us show (i). Taking the dual of $d s^{1}, \ldots, d s^{n}$, we obtain

$$
\frac{\partial}{\partial s^{\beta}}=\sum_{\gamma=1}^{n} X_{\beta}^{\gamma} \partial \gamma \quad(1 \leq \beta \leq n) .
$$

Applying $\nabla_{\partial_{\alpha}}^{c s}$, we have

$$
\nabla_{\partial_{\alpha}}^{c s} \frac{\partial}{\partial s^{\beta}}=\sum_{\delta=1}^{n}\left(\partial_{\alpha} X+\Upsilon_{\alpha} X\right)_{\beta}^{\delta} \partial_{\delta} \stackrel{(7.6)}{=} 0
$$

Thus $s=\left(s^{1}, \ldots, s^{n}\right)$ is a system of $\nabla^{c s}$-flat coordinates.

Finally we show (ii). Denote by $\hat{C}_{\alpha \beta}^{\gamma}$ the structure constants of $\circledast=*$ with respect to the new basis $\frac{\partial}{\partial s^{1}}, \ldots, \frac{\partial}{\partial s^{n}}$ :

$$
\frac{\partial}{\partial s^{\alpha}} * \frac{\partial}{\partial s^{\beta}}=\sum_{\gamma=1}^{n} \hat{C}_{\alpha \beta}^{\gamma} \frac{\partial}{\partial s^{\gamma}}
$$

Then by (7.8),

$$
\hat{C}_{\alpha \beta}^{\gamma}=\sum_{\mu, \nu, \lambda} X_{\alpha}^{\mu} X_{\beta}^{\nu} C_{\mu \nu}^{\lambda}\left(X^{-1}\right)_{\lambda}^{\gamma} .
$$

Since $X, X^{-1}, C_{\mu} \in M_{n}\left(\mathbb{C}\left[t^{\prime}\right]\right), \hat{C}_{\alpha \beta}^{\gamma} \in \mathbb{C}\left[t^{\prime}\right]=\mathbb{C}\left[s^{\prime}\right]$. Theorem 4.5 is proved.

\section{Proof of Theorem 5.5}

The notations are the same as $₫ 7$.

Lemma 8.1. $\nabla^{c s}=\nabla$ holds if and only if $A$ is an anti-diagonal constant matrix.

Proof. The condition $\nabla^{c s}=\nabla$ is equivalent to $\Upsilon_{\alpha}=O(1 \leq \alpha \leq n)$. Therefore by (7.4) $\nabla^{c s}=\nabla$ holds if and only if $A$ is a constant matrix. By Lemma 6.2, $A$ is a constant matrix if and only if it is anti-diagonal.

Remark 8.2. In the case $n=2, A$ is given by

$$
A=\frac{d_{2}-1}{d_{1}}\left(\begin{array}{cc}
0 & 1 \\
1 & C_{22}^{2}
\end{array}\right) \text {. }
$$

Therefore by Lemma 8.1, $A$ is an anti-diagonal if and only if $C_{22}^{2}=0$. It is not difficult to compute $C_{22}^{2}$. See [1, §5], [6, Tables C6, C7 ,C8]. Among exceptional Shephard groups of rank $2, C_{22}^{2} \neq 0$ holds only for $G_{4}, G_{5}, G_{8}, G_{16}, G_{20}$. This result agrees with Theorem 5.4 proved by the calculation using the $u$-coordinates.

Next we consider the metric $\eta$ defined by (4.10).

Lemma 8.3. The matrix A defined in (6.16) is the representation matrix of the metric $\eta$ with respect to $\partial_{\alpha}(1 \leq \alpha \leq n)$ :

$$
\eta\left(\partial_{\alpha}, \partial_{\beta}\right)=A_{\alpha \beta} \quad(1 \leq \alpha, \beta \leq n) .
$$


Proof. Substituting $\circledast=*, X=\partial_{\alpha}$ and $Y=\partial_{\beta}$ into (4.10), we obtain

$$
\eta\left(\partial_{\alpha}, \partial_{\beta}\right)=h\left(\partial_{\alpha}, E * \partial_{\beta}\right)=h\left(\partial_{\alpha}, \sum_{\gamma=1}^{n} U_{\beta}^{\gamma} \partial_{\gamma}\right)=(H U)_{\alpha \beta} .
$$

Since $A=H U$ (see Lemma 6.1),$\eta\left(\partial_{\alpha}, \partial_{\beta}\right)=A_{\alpha \beta}$.

Now we prove Theorem 5.5. Assume that $\theta$ is a metric on $M$ compatible with the natural Saito structure $(\nabla, *, E)$. Then $\theta$ must satisfy (2.5), (2.6) , (2.7) (with $\eta$ replaced by $\theta)$. Let $\Theta$ be the representation matrix of $\theta$ with respect to $\partial_{\alpha}(1 \leq \alpha \leq n)$, i.e.

$$
\Theta_{\alpha \beta}=\theta\left(\partial_{\alpha}, \partial_{\beta}\right)
$$

Then (2.5) is equivalent to

$$
\partial_{\alpha} \Theta_{\beta \gamma}=0 \quad(1 \leq \alpha, \beta, \gamma \leq n)
$$

Therefore $\Theta$ must be a constant matrix.

Eq.(2.7) is equivalent to

$$
\frac{d_{\alpha}+d_{\beta}}{d_{1}} \Theta_{\alpha \beta}=(2-D) \Theta_{\alpha \beta} \quad(1 \leq \alpha, \beta \leq n)
$$

Since $\Theta \neq O$, it follows that $2-D=\left(d_{\alpha}+d_{\beta}\right) / d_{1}$ must holds for some $(\alpha, \beta)$. Let us show that

$$
2-D=\frac{d_{1}+d_{n}}{d_{1}} .
$$

First assume that $d_{1}(2-D)>d_{1}+d_{n}$ holds. Then by (4.2),

$$
\frac{d_{\alpha}+d_{\beta}}{d_{1}} \leq \frac{d_{1}+d_{n}}{d_{1}}<2-D \quad(\alpha+\beta \geq n+1) .
$$

So $\Theta_{\alpha \beta}=0$ must hold for $\alpha+\beta \geq n+1$, which implies $\operatorname{det} \Theta=0$. This contradicts the nondegeneracy of the metric $\theta$. Therefore $d_{1}(2-D) \leq d_{1}+d_{n}$. By a similar argument, we can show $d_{1}(2-D) \geq d_{1}+d_{n}$. Thus we have

$$
2-D=\frac{d_{1}+d_{n}}{d_{1}}, \quad \Theta_{\alpha \beta}=0 \quad(\alpha+\beta \neq n+1), \quad \Theta_{\alpha, n+1-\alpha} \in \mathbb{C} \backslash\{0\} .
$$

Finally, the condition (2.6) implies $\theta\left(\partial_{\alpha}, \partial_{\beta}\right)=\theta\left(\partial_{\alpha} * \partial_{\beta}, \partial_{1}\right)$. So

$$
\Theta_{\alpha \beta}=\sum_{\gamma=1}^{n} C_{\alpha \beta}^{\gamma} \Theta_{\gamma, 1} \stackrel{\text { (8.1) }}{=} C_{\alpha \beta}^{n} \Theta_{n, 1} \stackrel{\text { Lemma } 6.1}{=} \frac{d_{1} \Theta_{n, 1}}{d_{n}-1} A_{\alpha \beta} .
$$

Since $\Theta$ is anti-diagonal, this equation implies that $A$ must be an anti-diagonal matrix. Hence by by Lemma 8.1, $\nabla=\nabla^{c s}$ must hold. Moreover $\theta$ must be a constant multiple of $\eta$ since $A$ is the representation matrix of $\eta$ (Lemma 8.3).

The proof of the converse is immediate. If we assume that $\nabla=\nabla^{c s}$, then it is clear that the constant multiple of $\eta$ is compatible with $\left(\nabla^{c s}, \circledast=*, E\right)$ since $\eta$ is a metric 
compatible with $\left(\nabla^{c s}, \circledast=*, E\right)$. (See the last paragraph in $\$ 4$.) This finishes the proof of Theorem 5.5.

\section{Appendix A. Vector Field e For $G(m, 1, n)$}

A.1. Preliminary. For a tuple of variables $v=\left(v^{1}, \ldots, v^{n}\right)$, let

$$
\mathbf{e}_{\alpha}(v)=\sum_{1 \leq i_{1}<i_{2}<\ldots<i_{\alpha} \leq n} v^{i_{1}} v^{i_{2}} \cdots v^{i_{\alpha}}
$$

be the $\alpha$-th elementary symmetric polynomial in $v$. We use the notation

$$
v_{(i, j, \ldots, k)}=v \backslash\left\{v^{i}, v^{j}, \ldots, v^{k}\right\}
$$

Notice that

$$
\mathbf{e}_{\alpha}\left(v_{(i, j, \ldots, k)}\right)=v^{l} \mathbf{e}_{\alpha-1}\left(v_{(i, j, \ldots, k, l)}\right)+\mathbf{e}_{\alpha}\left(v_{(i, j, \ldots, k, l)}\right) \quad(l \neq i, j, \ldots, k)
$$

We set

$$
\mathbb{E}(v)=\mathbb{E}\left(v^{1}, \ldots, v^{n}\right)=\left(\begin{array}{ccc}
1 & \ldots & 1 \\
\mathbf{e}_{1}\left(v_{(1)}\right) & \ldots & \mathbf{e}_{1}\left(v_{(n)}\right) \\
\vdots & & \vdots \\
\mathbf{e}_{n-1}\left(v_{(1)}\right) & \ldots & \mathbf{e}_{n-1}\left(v_{(n)}\right)
\end{array}\right) .
$$

Lemma A.1. The determinant of $\mathbb{E}(v)$ is given by

$$
|\mathbb{E}(v)|=\prod_{1 \leq k<l \leq n}\left(v^{k}-v^{l}\right)
$$

Proof. Subtracting the first column from the $k$-th column $(k>1)$ and using (A.1), we have

$$
\begin{aligned}
\left|\mathbb{E}\left(v^{1}, \ldots, v^{n}\right)\right|=\left|\begin{array}{cccc}
1 & 0 & \cdots & 0 \\
\mathbf{e}_{1}\left(v_{(1)}\right) & v^{1}-v^{2} & \cdots & v^{1}-v^{n} \\
\mathbf{e}_{2}\left(v_{(2)}\right) & \left(v^{1}-v^{2}\right) \mathbf{e}_{1}\left(v_{(1,2)}\right) & \cdots & \left(v^{1}-v^{n}\right) \mathbf{e}_{1}\left(v_{(1, n)}\right) \\
\vdots & \vdots & \vdots \\
\mathbf{e}_{n}\left(v_{(n)}\right) & \left(v^{1}-v^{2}\right) \mathbf{e}_{n-1}\left(v_{(1,2)}\right) & \cdots & \left(v^{1}-v^{n}\right) \mathbf{e}_{n-1}\left(v_{(1, n)}\right)
\end{array}\right| \\
=\prod_{2 \leq k \leq n}\left(v^{1}-v^{k}\right) \cdot\left|\mathbb{E}\left(v^{2}, \ldots, v^{n}\right)\right| .
\end{aligned}
$$

The claim follows by induction.

Next we calculate the $(\alpha, j)$ minor of $\mathbb{E}(v)$. Let $\mathbb{E}(v)_{\alpha, j}$ be the matrix obtained by deleting the $\alpha$-th row and the $j$-th column of $\mathbb{E}(v)$. 
Lemma A.2. The determinant of $\mathbb{E}(v)_{\alpha, j}$ is given by

$$
\left|\mathbb{E}(v)_{\alpha, j}\right|=\left(v^{j}\right)^{n-\alpha} \prod_{\substack{1 \leq k<l \leq n ; \\ k, l \neq j}}\left(v^{k}-v^{l}\right) .
$$

Proof. We may assume $j=1$, since

$$
\left|\mathbb{E}\left(v^{1}, v^{2}, \ldots, v^{n}\right)_{\alpha, j}\right|=(-1)^{j-2}\left|\mathbb{E}\left(v^{j}, v^{2}, \ldots, v^{j-1}, v^{1}, v^{j+1}, v^{n}\right)_{\alpha, 1}\right| .
$$

First we consider the case $\alpha=1$. Subtract the first column of $\mathbb{E}(v)_{1,1}$ from the $k$-th column $(k>1)$. Then subtract the second column from the $k$-th column $(k>2)$. Continuing this process, we have

$$
\begin{aligned}
& \left|\mathbb{E}(v)_{1,1}\right|=\left|\begin{array}{cccc}
\mathbf{e}_{1}\left(v_{(2)}\right) & \mathbf{e}_{1}\left(v_{(3)}\right) & \cdots & \mathbf{e}_{1}\left(v_{(n)}\right) \\
\mathbf{e}_{2}\left(v_{(2)}\right) & \mathbf{e}_{2}\left(v_{(3)}\right) & \cdots & \mathbf{e}_{2}\left(v_{(n)}\right) \\
\vdots & \vdots & \ddots & \vdots \\
\mathbf{e}_{n-1}\left(v_{(2)}\right) & \mathbf{e}_{n-1}\left(v_{(3)}\right) & \cdots & \mathbf{e}_{n-1}\left(v_{(n)}\right)
\end{array}\right| \\
& =\prod_{3 \leq k \leq n}\left(v^{2}-v^{k}\right)\left|\begin{array}{cccc}
\mathbf{e}_{1}\left(v_{(2)}\right) & 1 & \cdots & 1 \\
\mathbf{e}_{2}\left(v_{(2)}\right) & \mathbf{e}_{1}\left(v_{(2,3)}\right) & \cdots & \mathbf{e}_{1}\left(v_{(2, n)}\right) \\
\vdots & \vdots & \ddots & \vdots \\
\mathbf{e}_{n-1}\left(v_{(2)}\right) & \mathbf{e}_{n-2}\left(v_{(2,3)}\right) & \cdots & \mathbf{e}_{n-2}\left(v_{(2, n)}\right)
\end{array}\right| \\
& =\prod_{3 \leq k \leq n}\left(v^{2}-v^{k}\right) \prod_{4 \leq k \leq n}\left(v^{3}-v^{k}\right)\left|\begin{array}{ccccc}
\mathbf{e}_{1}\left(v_{(2)}\right) & 1 & 0 & \cdots & 0 \\
\mathbf{e}_{2}\left(v_{(2)}\right) & \mathbf{e}_{1}\left(v_{(2,3)}\right) & 1 & \cdots & 1 \\
\vdots & \vdots & \ddots & \vdots & \\
\mathbf{e}_{n-1}\left(v_{(2)}\right) & \mathbf{e}_{n-2}\left(v_{(2,3)}\right) & \mathbf{e}_{n-3}\left(v_{(2,3,4)}\right) & \cdots & \mathbf{e}_{n-3}\left(v_{(2,3, n)}\right)
\end{array}\right| \\
& \vdots
\end{aligned}
$$

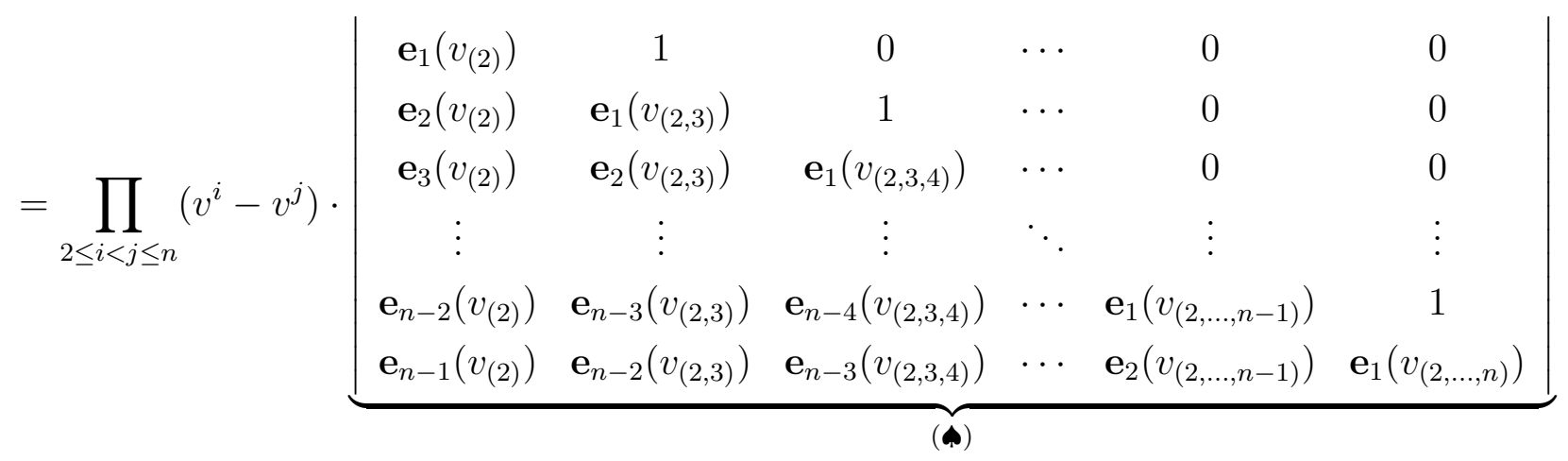

Next we eliminate the entries below the diagonal of $(\boldsymbol{\phi})$ by column operations. We start from the bottom row. For $k<n-1$, eliminate the $(n-1, k)$ entry $\mathbf{e}_{n-k}\left(v_{(2, \ldots, k+1)}\right)=$ $v^{1} v^{k+2} \cdots v^{n}$ using the $(n-1, n-1)$ entry $\mathbf{e}_{1}\left(v_{(2, \ldots, n)}\right)=v^{1}$. Then $(n-2, n-2)$ entry 
becomes $\mathbf{e}_{1}\left(v_{(2, \ldots, n-1)}\right)-v_{n}=v_{1}$ and all the $(n-2, k)$ entries for $k<n-2$ are divisible by $v_{1}$. Repeating the elimination process, we obtain

$$
(\boldsymbol{A})=\left|\begin{array}{cccc}
v^{1} & 1 & & O \\
& \ddots & \ddots & \\
& & v^{1} & 1 \\
O & & & v^{1}
\end{array}\right|=\left(v^{1}\right)^{n-1} .
$$

This proves the formula for $\alpha=1$. The cases $\alpha>1$ may be reduced to the case $\alpha=1$ as follows. By column operations similar to those in Lemma A.1, we have

$$
\begin{aligned}
\left|\mathbb{E}(v)_{\alpha, 1}\right| & =\prod_{2 \leq i \leq \alpha}\left(\prod_{i<j \leq n}\left(v^{i}-v^{j}\right)\right) \cdot\left|\begin{array}{ccc}
\mathbf{e}_{1}\left(v_{(2, \cdots, \alpha, \alpha+1)}\right) & \cdots & \mathbf{e}_{1}\left(v_{(2, \cdots, \alpha, n)}\right) \\
\vdots & \ddots & \vdots \\
\mathbf{e}_{n-\alpha}\left(v_{(2, \cdots, \alpha, \alpha+1)}\right) & \cdots & \mathbf{e}_{n-\alpha}\left(v_{(2, \cdots, \alpha, n)}\right)
\end{array}\right| \\
& =\prod_{2 \leq i \leq \alpha}\left(\prod_{i<j \leq n}\left(v^{i}-v^{j}\right)\right) \cdot\left|\mathbb{E}\left(v^{1}, v^{\alpha+1}, \ldots, v^{n}\right)_{1,1}\right|
\end{aligned}
$$

Thus the formula for $\alpha>1$ follows from that for $\alpha=1$.

From the above two lemmas, we obtain the following

Lemma A.3. The $(i, \alpha)$ entry of $\mathbb{E}(v)^{-1}$ is given by

$$
\left(\mathbb{E}(v)^{-1}\right)_{i \alpha}=(-1)^{\alpha+1}\left(v^{i}\right)^{n-\alpha} \prod_{\substack{1 \leq l \leq n ; \\ l \neq i}}\left(v_{i}-v_{l}\right)^{-1} .
$$

Proof. Substitute the formulas in Lemmas A.1 and A.2 into

$$
\left(\mathbb{E}(v)^{-1}\right)_{i \alpha}=\frac{(-1)^{i+\alpha}\left|\mathbb{E}(v)_{\alpha, i}\right|}{|\mathbb{E}(v)|}
$$

A.2. The vector field $e$ for $G(m, 1, n)$. If we set

$$
v^{i}=\left(u^{i}\right)^{m} \quad(1 \leq i \leq n),
$$

then a set of basic invariants for $G(m, 1, n)$ is given by

$$
x^{\alpha}=\mathbf{e}_{n+1-\alpha}(v) \quad(1 \leq \alpha \leq n) .
$$

Proposition A.4. We have

$$
\frac{\partial u^{i}}{\partial x^{\alpha}}=\frac{(-1)^{n+\alpha}\left(u^{i}\right)^{m(\alpha-2)+1}}{m} \prod_{\substack{1 \leq l \leq n \\ l \neq i}}\left(v_{i}-v_{l}\right)^{-1} .
$$


Proof. From (A.1), it is immediate to see that

$$
\frac{\partial}{\partial v^{i}} \mathbf{e}_{\alpha}(v)=\mathbf{e}_{\alpha-1}\left(v_{(i)}\right) .
$$

Therefore, using the chain rule, we have

$$
\begin{aligned}
\left(\begin{array}{ccc}
\frac{\partial x^{n}}{\partial u^{1}} & \ldots & \frac{\partial x^{n}}{\partial u^{n}} \\
\vdots & & \vdots \\
\frac{\partial x^{1}}{\partial u^{1}} & \ldots & \frac{\partial x^{1}}{\partial u^{n}}
\end{array}\right) & =\left(\begin{array}{ccc}
\frac{\partial x^{n}}{\partial v^{1}} & \ldots & \frac{\partial x^{n}}{\partial v^{n}} \\
\vdots & & \vdots \\
\frac{\partial x^{1}}{\partial v^{1}} & \ldots & \frac{\partial v^{1}}{\partial u^{n}}
\end{array}\right)\left(\begin{array}{ccc}
\frac{\partial v^{1}}{\partial u^{1}} & \ldots & \frac{\partial v^{1}}{\partial u^{n}} \\
\vdots & & \vdots \\
\frac{\partial v^{n}}{\partial u^{1}} & \ldots & \frac{\partial v^{n}}{\partial u^{n}}
\end{array}\right) \\
& =m \mathbb{E}(v) \operatorname{diag}\left(\left(u^{1}\right)^{m-1}, \ldots,\left(u^{n}\right)^{m-1}\right)
\end{aligned}
$$

Therefore by the inverse function theorem,

$$
\left(\begin{array}{ccc}
\frac{\partial u^{1}}{\partial x^{n}} & \ldots & \frac{\partial u^{1}}{\partial x^{1}} \\
\vdots & & \vdots \\
\frac{\partial u^{n}}{\partial x^{n}} & \ldots & \frac{\partial u^{n}}{\partial x^{1}}
\end{array}\right)=\frac{1}{m} \operatorname{diag}\left(\left(u^{1}\right)^{1-m}, \ldots,\left(u^{n}\right)^{1-m}\right) \mathbb{E}(v)^{-1}
$$

Comparing the $(i, n+1-\alpha)$ entries of the both sides, we obtain

$$
\frac{\partial u^{i}}{\partial x^{\alpha}}=\frac{\left(u^{i}\right)^{1-m}}{m}\left(\mathbb{E}(v)^{-1}\right)_{i, n+1-\alpha}
$$

Thus the statement follows from Lemma A.3.

Corollary A.5. The vector field $e=\frac{\partial}{\partial x^{1}}$ is given as follows.

$$
e=\sum_{k=1}^{n} e^{k} \frac{\partial}{\partial u^{k}}, \quad e^{k}=\frac{(-1)^{n+1}}{m\left(u^{k}\right)^{m-1}} \prod_{\substack{1 \leq l \leq n \\ l \neq k}}\left(v_{k}-v_{l}\right)^{-1}
$$

\section{REFERENCES}

[1] Arsie, Alessandro and Lorenzoni, Paolo, Complex reflection groups, logarithmic connections and bi-flat F-manifolds, Lett. Math. Phys. 107 (2017), no.10, 1919-1961.

[2] Bessis, David, Finite complex reflection arrangements are $K(\pi, 1)$, Ann. of Math. (2) 181 (2015), no. 3, 809-904.

[3] Dubrovin, Boris, Geometry of 2D topological field theories, in Integrable systems and quantum groups (Montecatini Terme, 1993), 120-348, Lecture Notes in Math. 1620, Springer, Berlin, 1996.

[4] _ On almost duality for Frobenius manifolds, in Geometry, topology, and mathematical physics, 75-132, Amer. Math. Soc. Transl. Ser. 2, 212, Amer. Math. Soc., Providence, RI, 2004.

[5] Kato, Mitsuo; Mano, Toshiyuki; Sekiguchi, Jiro, Flat structure on the space of isomonodromic deformations, arXiv:1511.01608v1 [math.CA].

[6] Konishi, Yukiko; Minabe, Satoshi; Shiraishi, Yuuki, Almost duality for Saito structure and complex reflection groups, J. Integrable Syst. 3 (2018), no. 1, xyy003, 48 pp.

[7] Lehrer, Gustav I. and Taylor, Donald E., Unitary reflection groups, Australian Mathematical Society Lecture Series, 20. Cambridge University Press, Cambridge, 2009. viii+294 pp.

[8] Maschke, Heinrich, Aufstellung des vollen Formensystems einer quaternären Gruppe von 51840 linearen Substitutionen, Math. Ann. 33 (1889), no. 3, 317-344. 
[9] Orlik, Peter and Terao, Hiroaki, Arrangements of hyperplanes, Grundlehren der Mathematischen Wissenschaften 300. Springer-Verlag, Berlin, 1992. xviii+325 pp.

[10] Sabbah, Claude, Déformations isomonodromiques et variétés de Frobenius, EDP Sciences, Les Ulis; CNRS Éditions, Paris, 2002. xvi+289 pp.

[11] Saito, Kyoji; Yano, Tamaki; Sekiguchi, Jiro, On a certain generator system of the ring of invariants of a finite reflection group, Comm. Algebra 8 (1980), no. 4, 373-408.

[12] Saito, Kyoji, On a linear structure of the quotient variety by a finite reflexion group, Publ. Res. Inst. Math. Sci. 29 (1993), no. 4, 535-579. (Preprint version: RIMS-288, Kyoto Univ., Kyoto, 1979.)

[13] Shephard, G. C. and Todd, J. A., Finite unitary reflection groups, Canadian J. Math. 6 (1954). 274-304.

Department of Mathematics, College of Liberal Arts, Tsuda University, Toyko 1878577, JAPAN

E-mail address: konishi@tsuda.ac.jp

Department of Mathematics, Tokyo Denki University, Tokyo 120-8551, Japan

E-mail address: minabe@mail.dendai.ac.jp 